\title{
LA COMPOSICIÓN NOMINAL EN ESPAÑOL: PROPUESTA DE CLASIFICACIÓN
}

\author{
Juan Manuel Pérez Vigaray \\ Universidad de Las Palmas de Gran Canaria \\ José Juan Batista Rodríguez \\ Universidad de La Laguna-Instituto Universitario de Lingüística Andrés Bello
}

\section{RESUMEN}

El presente trabajo trata de delimitar el concepto de compuesto nominal dentro del campo más amplio de las palabras compuestas en español. Nos centramos concretamente en el estudio de los llamados compuestos léxicos, y, a partir de un análisis crítico de las clasificaciones más conocidas de los últimos 30 ańos para nuestra lengua, proponemos una nueva clasificación que toma como criterio rector la relación gramatical que se establece entre los miembros constituyentes del compuesto y se aplica desde el primer nivel clasificatorio. Se abandona, así, la línea más seguida en español, que suele tomar como criterio rector de sus clasificaciones, en el primer y en el segundo nivel clasificatorio, la categoría gramatical, ya sea la categoría final del compuesto o la categoría de los elementos constituyentes. Después de describir brevemente cada uno de los tipos que proponemos, la segunda parte del trabajo se centra en poner de manifiesto la importancia de los aspectos diacrónicos y tipológicos en el estudio de los compuestos españoles (y románicos) y en nuestra propuesta de clasificación. Palabras Clave: formación de palabras, composición nominal, morfología, semántica.

\section{SPANISH COMPOUNDING: A NEW PROPOSAL OF CLASSIFICATION}

\section{Abstract}

This study aims to delimit the concept of noun compound within the largest realm of Spanish compound words. We are specifically focusing on the so called lexical compounds and, from a critical analysis of the most extended classifications for our language in the last 30 years, we bring forward a new classification that takes the grammatical relationship established between the components of the compound as the leading criterion which is applied from the first classification level. Thus, we abandon the most extended research approach in Spanish, which usually takes the grammatical category as the organizing criterion of its classifications, on the first and second classification levels, whether they are the resulting compound category or the category of the compound components. After a brief description of each of the types presented, the research focuses on highlighting the importance of the diachronic and typological issues in the study of Spanish, and also of Romance compounds as well as in our own classification.

KEYWORDS: word formation, nominal compounding, morphology, semantics.

DOI: https://doi.org/10.25145/j.refiull.2020.40.11

Revista de Filología, 40; enero 2020, pp. 205-245; ISSN: e-2530-8548 


\section{PALABRAS COMPUESTAS EN ESPAÑOL}

La primera cuestión que se plantea a la hora de abordar el estudio de la composición nominal en cualquier lengua indoeuropea es de qué estamos hablando cuando empleamos el término palabra compuesta. E, inmediatamente, surgen las siguientes preguntas: ¿¿s igual palabra compuesta que compuesto nominal? ¿Constituye la composición nominal un procedimiento de formación de palabras paralelo a la derivación? ¿Los criterios a los que hemos de acudir preferentemente para su descripción y clasificación serán de tipo morfológico, semántico o sintáctico?

A pesar de que en los últimos 30 años ha habido una eclosión de estudios sobre la formación de palabras en español y, más en concreto, sobre la composición nominal, todavía no podemos decir que haya consenso sobre las respuestas a las preguntas que acabamos de plantear. Y en esta misma situación se encuentran tanto los estudios centrados en lenguas concretas como los de orden más general y tipológico. En este sentido, las siguientes palabras de Bauer (2017: 2) son, sin duda, perfectamente aplicables a los trabajos sobre composición de palabras en español:

Even the question of whether compounding is a morphological or a syntactic one is no settled. [...] There is huge disagreement about the classification of compounds. [...] And there is certainly disagreement on where the boundaries of compounding are to be found and about the terminology of compounding.

Sin entrar en la discutidísima definición de palabra compuesta (Batista 1988: 61-140; Rainer / Varela 1992: 117-124; Pérez Vigaray 2004: 42-92; Ralli 2013: 25, 47, 49-51, 57-58, 76-79, etc.; Tribulato 2015: 13-61; Booij, 201233: 77-98 y Bauer 2017: 3-28) ${ }^{1}$, nos conformamos con precisar que se considera tal al resultado de la fusión de dos lexemas independientes (temas o palabras) ${ }^{2}$, con los que suele presen-

${ }^{1}$ Sobre si la prefijación debe considerarse dentro de la derivación o dentro de la composición de palabras se ha escrito tanto que no merece la pena insistir: nos limitamos a constatar que a lo largo del siglo xx se ha venido incluyendo en la derivación. También se ha escrito mucho sobre la distinción entre compuesto y fraseologismo: $c f$. Pérez Vigaray / Batista 2005, Montoro del Arco 2008, García Padrón / Batista 2010, etc. En este aspecto coincidimos con la main stream espańola, representada por Rainer / Varela (1992: 117-122), quienes niegan el carácter de verdaderos compuestos tanto a los verbos, sustantivos y adjetivos con prefijo (Rainer / Varela 1992: 118 y 122) como a los sintagmas del tipo de telón de acero, media luna, luna nueva (Rainer / Varela 1992: 120), aunque incluyen dentro de la composición a los llamados compuestos coordinativos, como clérigo poeta (Rainer / Varela 1992: 119).

2 Cf. Ralli (2013: 76): «With some exceptions, the first constituent of a compound is usually a stem, while the second is a stem or a word». Lo mismo afirma Tribulato (2015: 18): «Greek compounds typically involve a FC [first constituent] that does not correspond to a full "word" but to a stem, and a SC [second constituent] which may consist of either a stem or an independently attested word». Como resumen de los problemas para definir palabra compuesta, cf. Lieber / Štekauer (2009: 4-14). Por lexemas entendemos las palabras lexemáticas de Coseriu (1978: 133), esto es, sustantivos, adjetivos y verbos, que coinciden con las que componen el Symbolfeld o campo simbólico del lenguaje de Bühler (1979: 167-273). Por esta razón excluimos, por ejemplo, a los compuestos con pre- 
tar diferencias morfosemánticas, y que la composición suele limitarse a la nominal $^{3}$. Así, las palabras compuestas constituyen, en español, un conjunto heterogéneo, en el que podemos hablar de -al menos- seis grandes grupos: a) en primer lugar, los compuestos léxicos del tipo lavaplatos, bocacalle, carricoche, pelirrojo, rojiblanco, maniobrar, malvender, b) en segundo lugar, los compuestos sintagmáticos del tipo teléfono móvil, media naranja, mano de obra, tomar el pelo, estirar la pata; c) en tercer lugar, los compuestos fraseales o frásticos, como hazmerreír, bienmesabe, nomeolvides, correveidile; d) en cuarto lugar, los compuestos por derivación, como ropavejero, centrocampista, sietemesino; e) en quinto lugar, los compuestos grecolatinos (cultos) del tipo de filología, ecografía, plenilunio, hipódromo, discoteca, etc., entre los que cabría incluir algunos compuestos artificiales ${ }^{4}$ del español, tomados como préstamos directos del latín o montados sobre el esquema latino, como terrateniente, grandilocuente, altitonante, drogodependiente o misacantano; f) y, por último, podría incluirse un sexto tipo constituido por aquellas palabras compuestas que reflejan esquemas o patrones compositivos tan ajenos al español como propios de la lengua de la que proceden estas formaciones: es el caso de tantos términos «traducidos», como sucede, por ejemplo, con teleserie, puticlub, trabajoadicto, piano bar, etc., montados sobre los patrones típicos del inglés tv serie, night club, workaholic, piano $b a r$, etc., por lo que, en cierto modo, constituyen una suerte de calco y, en definitiva, de préstamo léxico. En suma, aunque no todas las voces citadas se hayan formado mediante un proceso de composición sensu stricto, lo cierto es que todas ellas son palabras "compuestas»".

Por nuestra parte, en esta ocasión acotaremos nuestro objeto de estudio al primer grupo, al de los compuestos léxicos.

fijo, ya que estos forman parte de las palabras morfemáticas de Coseriu y el Zeigfeld o campo mostrativo de Bühler.

3 Siguiendo una tradición que remonta a Franz Bopp, Risch $\left(1974^{2}: 181\right)$ afirma: «Alle echten Komposita der griechischen Sprache sind Nomina». Confirman esta tesis para el griego antiguo, entre otros autores, Meissner y Tribulato (2002: 301) y Ralli (2009: 453). Para los compuestos

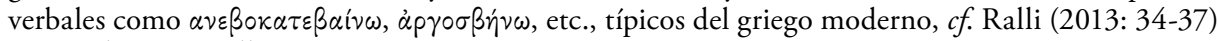
y Manolessou / Ralli (2015: 2056-2057).

${ }^{4}$ Palabras compuestas que ya, en el propio latín, eran igualmente artificiales, en muchos

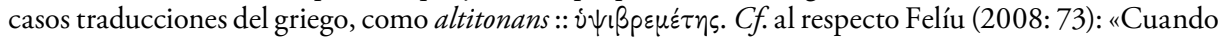
el orden de los elementos del compuesto contraviene el orden habitual de la sintaxis, como en los casos de complemento núcleo (maniatar, perniquebrar, terrateniente), suele tratarse de esquemas heredados del latín, poco productivos en español actual».

5 Ya Gauger (1971: 164) hablaba de cinco tipos principales de compuestos en español, que ejemplificaba como sigue: 1 . perro de caza; 2 . hombre-masa; 3 . autoescuela; 4. sacacorchos; y 5 . caritriste. 


\section{COMPUESTOS LÉXICOS Y COMPUESTOS SINTAGMÁTICOS}

Actualmente, la práctica totalidad de los trabajos sobre composición nominal en español establecen una primera distinción entre dos grandes grupos o tipos de compuestos, que, según Val Álvaro (1999: 4759), «no son de la misma naturaleza», a saber: los compuestos léxicos y los compuestos sintagmáticos.

Los léxicos (Val Álvaro 1999, Felíu 2008) reciben también otras denominaciones: propios (Bustos 1986, Val Álvaro 1999), ortográficos o perfectos (Val Álvaro 1999), yuxtapuestos (Almela 1999), etc. Se caracterizan por la amalgama fónica de sus miembros con su correspondiente reflejo ortográfico, por la presencia de un solo acento principal y por la juntura morfemática. Según Val Álvaro (1999: 4759), un compuesto léxico o propio es una "palabra cohesionada morfológica, fonológica y ortográficamente. [...] Es palabra fonológica y unidad morfológica plena». Y, según este mismo autor (Val Álvaro 1999: 4760), en los compuestos léxicos se da un proceso de composición que opera sobre palabras y que consiste en la combinación de dos palabras para crear una palabra nueva.

Por su parte, los sintagmáticos conforman el segundo gran grupo de compuestos españoles. Este término fue introducido en la gramaticografía española por Eugenio de Bustos en 1986 y se ha mantenido hasta hoy, aceptado por prácticamente todos los estudiosos que desde entonces se han ocupado de la composición de palabras en español. Los compuestos sintagmáticos son «aquellos que, en su estructura formal, no ofrecen diferencia alguna respecto a los sintagmas nominales, constituidos de dos sustantivos unidos por medio de la preposición de, o por un sustantivo y un adjetivo» (Bustos 1986: 69). Para Val Álvaro (1999: 4760), en los compuestos sintagmáticos actúa un "proceso de reinterpretación que opera sobre construcciones sintácticas [...] Resulta de la fijación de una estructura sintáctica en una forma determinada, lo que conlleva la pérdida de propiedades sintácticas y la hace hábil para expresar conceptos unitarios».

A pesar del casi total acuerdo en la existencia y consideración de estos dos tipos, encontramos diferencias más o menos importantes entre los distintos estudiosos, sobre todo en lo que respecta a las fronteras existentes entre ellos. Sin embargo, tampoco faltan trabajos (Rainer y Varela 1992, Pérez Vigaray / Batista Rodríguez 2005, García Padrón / Batista Rodríguez 2010, etc.) en que, denunciando la incongruencia de considerar compuestos a los sintagmáticos ${ }^{6}$, se les relega a un ámbito periférico cercano a la fraseología.

${ }^{6}$ Cf. Booij (201233: 85-86): "Certain lexical expressions in Romance languages are sometimes incorrectly called compounds although they have in fact a phrasal form. This applies to French salle à manger 'dining room' and chambre d'hôtes 'guest room'. The structures $N$ à $N$ and $N$ de $N$ are instantiations of the syntactic structure $[\mathrm{N} \mathrm{PP}]_{\mathrm{NP}}$, a noun phrase consisting of a head $\mathrm{N}$ followed by a PP complement, and have developed into constructional idioms. Such phrases are functionally equivalent to compounds in Germanic languages, and that is why the mistake is made to consider them compounds. Note, however, that their plural forms are salle-s à manger and chambre-s d'hôtes respectively, with an internal plural ending. This proves their phrasal nature since the plural form of 
Por nuestra parte, en esta ocasión nos centraremos en los compuestos léxicos, los únicos que, entre otras características, permiten sostener la tan repetida afirmación de que «la composición de palabras en español, como en las lenguas romances en general, es un proceso menos productivo que en otras lenguas, por ejemplo en las germánicas» (Val Álvaro 1999: 4759). En efecto, si a los compuestos léxicos añadiéramos los sintagmáticos, el español y las demás lenguas romances tendrían un sistema compositivo tan productivo como el de las lenguas germánicas. Como veremos, esto sucede porque las lenguas romances no hacen más que seguir los patrones latinos de formación de palabras, en los que, como es de sobra sabido, primaba la derivación sobre la composición: desde Meillet / Vendryes (19795:420421) viene repitiéndose sin cesar que la traslación latina de los compuestos griegos

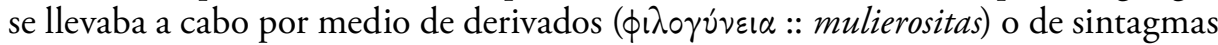

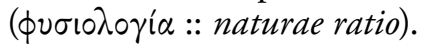

\section{CLASIFICACIONES DE LOS COMPUESTOS LÉXICOS ESPAÑOLES}

A continuación, y tras revisar catorce de las muchas clasificaciones de los compuestos españoles que se han propuesto lo largo de los últimos treinta ańos, analizaremos los criterios clasificatorios en los que se basan 7 . Como suele repetirse, los criterios clasificatorios empleados en estas clasificaciones son tanto morfológicos como sintácticos, semánticos y pragmáticos, todos ellos en intrincada relación, pues a menudo se combinan, implican y solapan. Por ello hay que distinguir niveles clasificatorios (hemos contado hasta cuatro): nuestro trabajo se centrará en los dos primeros niveles.

\subsection{LA CATEgORÍA GRAMATICAL}

El criterio clasificatorio más frecuentemente usado en español es el categorial, bien el de la categoría final del compuesto, bien el de las categorías de sus miembros. Así, lo habitual es que, en el primer nivel de clasificación, se acuda al criterio de la categoría final del compuesto, distinguiéndose de este modo compuestos sustantivos, compuestos adjetivos y compuestos verbales (no nos referimos aquí a los llamados verbos compuestos por prefijos, que desde el primer tercio del siglo xx se incluyeron en la prefijación; $c f$. Lindner 2011: 36-38). Sin embargo, no todos los

\footnotetext{
a French word is expressed by a suffix at its right edge. Another type of apparent French compound is homme-grenouille 'lit. man frog, frogman'. Its plural form requires both constituents to be pluralized (hommes-grenouilles) which suggest that we have to do with an NP in which the phrasal head is followed by a noun with an appositional function».

${ }^{7}$ Las clasificaciones que hemos analizado son las de Bustos (1986), Varela (1990a), Lang (1992), Rainer (1993), Pérez Vigaray (1994), Almela (1999), Val Álvaro (1999), Buenafuentes (2007), Felíu (2008), la NGLE (2010), Moyna (2011), Hernández / Marqueta (2015) y Fábregas (2015).
} 
estudiosos aceptan la existencia de compuestos verbales, de manera que la inclusión o exclusión de verbos compuestos como maniatar, maldecir o bienpensar constituye una de sus principales y más importantes diferencias en estas tipologías. En este sentido, las clasificaciones de Bustos (1986), Lang (1992), Pérez Vigaray (1994) Almela (1999) y Fábregas (2015) no incluyen compuestos verbales, mientras que las de Varela (1990), Rainer (1993), Val Álvaro (1999), Buenafuentes (2007), Felíu (2008), la NGLE (2010) y Moyna (2011) incluyen verbos compuestos (tanto los del tipo maniatar como los del tipo malpensar o bienquerer) $)^{8}$.

De la rareza de los compuestos verbales en español (y otras lenguas romances) dan cuenta tanto Hernández / Marqueta (2015) como Fábregas (2015: 253), quien habla de «formaciones casi excepcionales, como man-i-atar, pero que pueden documentarse en inglés y muchas otras lenguas germánicas». En griego moderno, que -como el antiguo- es una lengua que cuenta con numerosos compuestos y esquemas compositivos diferentes, hay compuestos verbales coordinativos (del tipo

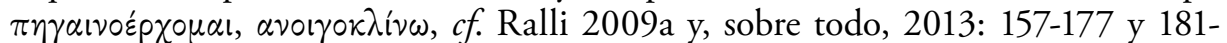
198) y se ha llegado incluso a hablar de compuestos verbales exocéntricos (Ralli 2013: 182-183)'.

Lo cierto es que, a pesar de que se tiende cada vez más a establecer tres categorías verbales de compuestos en las lenguas indoeuropeas actuales (sustantivos, adjetivos y verbos), todavía se mantiene con fuerza la idea de que la composición indoeuropea -tanto antigua como moderna- es propiamente nominal, no verbal, ya que los compuestos verbales resultan, en general, de procesos de derivación y parasíntesis ${ }^{10}$. Bástenos como ejemplo una cita de Ernst Risch (1974²: 181-182), afamado estudioso de la formación de palabras en Homero, al que siguen teniendo en cuenta todos los que se ocupan de la composición de palabras tanto en griego antiguo como moderno ${ }^{11}$ :

${ }^{8}$ Y ello, repetimos, desde el primer nivel de clasificación. Por otra parte, es interesante destacar que todas las clasificaciones que contemplan los verbos compuestos los incluyen como un tipo más, mientras que la RAE/ASALE los inserta en el cajón de sastre «Otras pautas de composición menos productivas".

9 Cf. Andreou (2015: 271-272): «That the creation of exocentric compounds in Greek and its dialects is a very prolific process is evident in the presence of verbs which are based on non-attested compounds. Consider the following examples: varikartizo, kalozoizo, kakoniktizo, kakoreksizo, kalostratizo...». Sobre esta cuestión vid. también Batista (2016) y Batista / Mora / Pérez Vigaray (2019).

${ }^{10} C f$. Bauer (1917: 51, 95, 99 y 136, respectivamente): «Marchand's (1969: 100) statement that '[v]erbal composition does not exist in Present-Day English' is possible only because an item like stagemanage is not created by modifying manage by stage, but by back-formation from stagemanager»; «The delimitation of compound verbs in the literature is not particularly clear»; «A common comment on verb compounds is that they are somehow rare or restricted»; y «Marchand's (1969) claim that English does not have compound verbs has gained a certain currency in the literature».

${ }^{11}$ En esta línea, Tribulato (2015: 126-128) también considera que, en griego antiguo, no había verbos compuestos, sino derivados mediante preverbios $(\dot{\varepsilon} \pi \iota \gamma \rho \alpha ́ \phi \omega)$ o por parasíntesis $(\varepsilon \dot{\varepsilon} \varepsilon p \gamma \varepsilon \tau \dot{\varepsilon} \omega)$. De la existencia de verbos compuestos en griego moderno (Ralli 2013: 157-175) trataremos en el apartado tipológico. 
Alle echten Komposita der griechischen Sprache sind Nomina; eigentliche zusammengesetzte Verben kennt sie nicht. Die mit Präpositionen (Lokaladverbien) zusammengesetzten Verben sind als Zusammenrückungen zu betrachten.

Por nuestra parte, y sin entrar a discutir este problema en la presente ocasión, estamos de acuerdo con considerar, en español, compuestos léxicos propios sólo a los nominales, ya que, en efecto, los llamados compuestos verbales en nuestra lengua resultan bien de parasíntesis (maniatar, perniquebrar), bien de anteponer a verbos simples los adverbios bien y mal (sobre todo), que funcionan más bien a modo de prefijos gramaticales (Buenafuentes 2001-2002).

En un segundo nivel de clasificación se aplica el criterio de la categoría de los miembros constituyentes, distinguiéndose, por ejemplo, entre los compuestos sustantivos los formados por sustantivo + sustantivo, sustantivo + adjetivo, adjetivo + sustantivo, etc., tal como hacen Bustos (1986), Varela (1990), Rainer (1993), Val Álvaro (1999), Buenafuentes (2007) y Felíu (2008). Pero no faltan los casos en que este criterio se aplica en el primer nivel: $c f$., por ejemplo, la clasificación de la NGLE (2010).

En nuestra opinión, el punto de vista categorial puede suponer un tamiz muy grueso en los dos primeros niveles clasificatorios, pues, por un lado, agrupa en un mismo tipo formaciones gramatical y semánticamente muy distintas entre sí; así, por ejemplo, bajo el mismo tipo «adjetival» se incluyen pelirrojola, anglohablante, vasodilatadorla y rojinegrola. Y, por la misma razón, entre los compuestos sustantivos se incluyen formaciones tan diferentes como guardabosques y bocamanga. Y, por otro lado, este criterio separa a veces compuestos que, sin embargo, pertenecen a un mismo y único tipo: tal ocurre con pelirrojo y rabigalgo, que, aunque se incluyan respectivamente en los tipos $[\mathrm{NiA}]$ y $[\mathrm{NiN}]$, no son dos invariantes semánticas diferentes, sino la misma, pues ambos son compuestos adjetivos formados por un primer miembro sustantivo, que es el elemento nuclear, regente o determinado, y un segundo miembro, que puede ser adjetivo (es lo más normal) o sustantivo, que es el elemento determinante o regido del compuesto. En efecto, en ambas variantes morfológicas de estos adjetivos compuestos se dan las mismas relaciones morfosemánticas: el segundo miembro determina al primero, que es siempre un sustantivo, de tal manera que el resultado final es un adjetivo interpretable como 'de X (primer miembro sustantivo) determinado por Y (adjetivo o sustantivo)'. Así, pelirrojo es un adjetivo que significa 'de pelo rojo' y rabigalgo es un adjetivo que significa 'de rabo de galgo'. Claro que el primero nos resulta normal, mientras que el segundo sorprende un poco: sólo lo encontramos en el Libro de Buen Amor (estr. 1219), pero su carácter de compuesto adjetivo es indiscutible. Más difícil, y a menudo mal entendido, resulta el adjetivo compuesto cachicuerno, predicado, por ejemplo, de cuchillos, con el significado 'de cachas de cuerno'. Y más raros aún -por tener respectivamente un segundo miembro sustantivo en - $a$ y una terminación poco frecuente que impiden la variación de género posible todavía en rabigalgo o cachicuerno (navajita cachicuerna) - resultan los adjetivos compuestos pelicabras y patibueyes, con los que, en La hora de todos, Quevedo califica al séquito de diosecillos que acompañan a Pan, pues aquí sólo es posible la variación de número. Pero esta misma dificultad, a la 
que se suma la usual imposibilidad de distinguir el plural, se advierte en los adjetivos verbonominales del tipo trotaconventos o tragaperras (Tabares / Pérez Vigaray / Batista 2010; Tabares / Batista 2013), los cuales, a juicio de Lüdtke (2011[2005]: 403), se originan, en principio, como adjetivos compuestos.

Por último, cualquier clasificación de corte categorial ha de tener en cuenta, además de todo lo expuesto, los posibles casos de "lexicalización», de modo que, por ejemplo, petirrojo o tragaldabas suelen considerarse normalmente sustantivos, obviando el hecho de que son meras sustantivaciones de adjetivos.

\subsection{LA RELACiÓN ENTRE LOS MiEMBROS DEL COMPUESTO}

Un criterio que, en los dos primeros niveles de las clasificaciones de compuestos españoles, ha sido poco empleado es el que atiende a la relación semántico-gramatical entre los miembros de un compuesto, relación que, a nuestro juicio, constituye el núcleo de su significado y, por tanto, resulta imprescindible para clasificarlo. En este sentido, de todas las clasificaciones que conocemos sólo se basan prioritariamente en este criterio la de Pérez Vigaray (1994), a quien sigue Almela (1999), la de Hernández / Marqueta (2015) y la de Fábregas (2015) ${ }^{12}$.

La relación semántica entre los miembros del compuesto es fundamental porque constituye su significado, al que no se puede llegar sólo desde la forma, esto es, atendiendo únicamente a la categoría de sus miembros constituyentes. De esta manera, dos compuestos como pelicabra y carricoche son, atendiendo a la categoría de sus miembros, iguales: se trata de compuestos formados por dos sustantivos unidos por la vocal - $i$-. Sin embargo, son muy distintos si atendemos a la relación semántica entre sus miembros, pues en pelicabra, como en patibuey o en rabigalgo, el primer miembro sustantivo, que es el elemento nuclear o regente del compuesto, está determinado por el segundo miembro sustantivo, que es el elemento regido, de manera que, en los tres casos, estamos ante compuestos de categoría adjetiva que se entienden como 'de pelo[s] de cabra', 'de pata[s] de buey' y 'de rabo de galgo' ${ }^{\prime 3}$. En cambio, en el caso de carricoche, baciyelmo o capisayo tenemos, como en el caso anterior, dos sustantivos unidos por la vocal -i-, pero no hay relación de determinación entre ambos miembros: el segundo miembro sustantivo no determina al primero, sino que ambos se encuentran al mismo nivel en una suerte de coordinación. Por esta razón se ha llamado a estos compuestos dvandva, copulativos o, más recientemente, coordinativos, si bien dentro de ellos es posible establecer subdivisiones ulte-

12 En efecto, la mayoría de las clasificaciones aplican este criterio sólo a partir del tercer nivel y, sobre todo, para subclasificar a los compuestos sustantivos del tipo sustantivo + sustantivo.

13 Por supuesto, como ha puesto de relieve Coseriu (1978: 245-246), siguiendo sobre todo a Morciniec (1964: 16), se trata de una relación general de determinación en el sistema lingüístico, que suele fijarse en la norma por medio de designaciones habituales. 
riores ${ }^{14}$. Así, dentro de estos compuestos coordinativos se distinguen formalmente aquellos que muestran una clara y coordinativa, como quitaipón, vaivén, tuiyó, calicanto, de los que presentan una - $i$ - «aprovechada», por llamar así a la que, perteneciendo a la raíz, se usa como vocal de unión, como vemos en agridulce o credivuelo. Y, aunque desde Munthe (1889 y 1901) y Baist (1899) hasta Moyna (2011: 225241) se ha discutido si es "compositiva» o "coordinativa» la - $i$ - de sustantivos como ajiaceite, ajipuerro, arquibanco, artimaña, capigorra, capisayo, carricoche, cervicabra, gallipavo, pasitrote, sopicaldo, tripicallos, etc., y de adjetivos como agridulce, altibajo, anchicorto, etc., lo cierto es que, atendiendo a la relación entre los miembros y a la categoría final del compuesto, en todos estos casos se trata de compuestos coordinativos, en cuya formación, como quiere Munthe (1901: 68-69), es muy posible que haya influido la - $i$ - compositiva latinizante que se introdujo "masivamente» en español en el siglo XV: de hecho, hay casos en que se documentan, por ejemplo, capa sayo y capisayo, por un lado, y agrodulce, agredulce y agriodulce, por otro (Moyna 2011: 228 y 239-240, respectivamente), siendo más antiguas las formaciones sin -i-, que, además, son las más normales en todas las lenguas románicas.

Por otra parte, los compuestos del tipo pelirrojo y patibuey se diferencian de carricoche y agridulce en que, en el primer caso, se trata de compuestos posesivos exocéntricos $\mathrm{y}$, en el segundo, de compuestos determinativos endocéntricos, rasgos en los que nos detendremos en el siguiente epígrafe y en el apartado tipológico de nuestro estudio, por lo que aquí nos contentaremos con señalar dos aspectos: el primero es que, en el caso de pelirrojo y patibuey, hay un miembro que es nuclear, regente o determinado y otro que es regido o determinado, mientras que, en el caso de carricoche y agridulce, no encontramos ninguna relación de determinación, pues los dos miembros se encuentran al mismo nivel. El segundo aspecto destacable es que, en el caso de pelirrojo y patibuey, la categoría final del compuesto es distinta a la de su elemento nuclear, ya que pelo y pata son sustantivos, mientras que pelirrojo y patibuey son adjetivos. En cambio, en el caso de carricoche y agridulce los dos miembros pertenecen a la misma clase de palabras, ya que, en el primer caso, son los dos sustantivos y, en el segundo, los dos son adjetivos; y, además, no se produce ningún cambio de categoría verbal, pues el compuesto pertenece a la misma clase de palabras que sus dos constituyentes: sustantivo, en el caso de carricoche, y adjetivo, en el caso de agridulce. Se observa, pues, que a la diferencia semántica entre estos dos grupos de compuestos se añaden también diferencias morfológicas que nos permiten distinguirlos. En suma, desde el punto de vista morfológico, los únicos compuestos que podrían confundirse son el tipo muy poco frecuente patibuey con el tipo, también poco frecuente, carricoche, ya que ambos presentan dos miembros sustantivos: sin embargo, se distinguen tanto por la existencia, en el primer caso, y la no exis-

${ }^{14}$ Así, por ejemplo, Gauger (1971: 140-141), siguiendo a Rohrer (1977[1965]), distingue tres subtipos de compuestos dvanda, copulativos o coordinativos: 1 . 'un X qui est á la fois A et B' (copier-duplicateur, mixeur-batteur, clair-obscur, doux-amer, aigredoux); 2. 'un A qui est aussi B' (robe-manteau, Homme-Dieu, café-bar); y 3. 'un A qui est un B' (voiture-restaurant, roi-enfant, enfantroi). Cf. también Bauer (2017: 82-93) 
tencia, en el segundo, de la relación de determinación como por categoría final del compuesto, adjetivo en el primer caso y sustantivo en el segundo, además de por el hecho bien conocido de que el tipo patibuey suele presentar el rasgo que los cognitivistas llaman 'posesión inalienable' y que, tradicionalmente, se ha expresado como que el primer miembro sustantivo de estos compuestos suele designar una parte del cuerpo (humano y animal) o una prenda de vestir ${ }^{15}$.

Acabaremos este epígrafe insistiendo en la importancia fundamental de la relación semántica entre los miembros del compuesto, ya que, en algunos (pocos) casos, la simple morfología no nos sirve de ayuda: tal ocurre, por ejemplo, con burriciego. En burriciego, adjetivo aplicado en principio a los toros que ven mal de cerca $y$, por tanto, suponen un gran peligro para el torero, la relación entre los miembros del compuesto no corresponde a ninguno de los tres posibles modelos de interpretación que describe García Lozano (1993[1978]: 210) a propósito de los compuestos del tipo pelirrojo, a saber: «1) ojinegro $\rightarrow$ que tiene los ojos negros, también con la variante pelirrojo $\rightarrow$ que tiene rojo el pelo. II) alirrojo $\rightarrow$ de alas rojas. III) carirredondo $\rightarrow$ redondo de cara». Sin embargo, como reconoce el propio García Lozano (1993[1978]: 210), «todas las realizaciones posibles se reducen a un módulo semántico, según el cual el segundo miembro predica una cualidad del primero». En efecto, los dos primeros tipos que establece García Lozano a partir de las definiciones del diccionario se reconducen sin ninguna dificultad a un mismo y único tipo (bien 'de ojos negros', bien 'que tiene las alas rojas') ${ }^{16}$, siendo que el tercero también se explica mejor como 'de cara redonda' o 'que tiene la cara redonda' que como 'redondo de cara', paráfrasis explicativa que resulta difícil de aplicar a la mayoría de los compuestos de este tipo ${ }^{17}$. En burriciego, la relación semántica que se establece entre ambos

15 Con mucha gracia responde Munthe (1901: 63) a la crítica que le había hecho Baist (1899) por no haber tenido en cuenta esta característica: «Ein weiterer mangel meiner untersuchung liege, nach Baist, in der definition: "composés d'un substantif et d'un adjectif, celui-ci déterminant celui-là, et unis à l'aide de la voyelle de liaison -i-". Dies sei wohl richtig, aber nicht genug, sagt Baist; ich hätte auch angeben sollen, dass es sich nicht um ein substantiv schlechthin handelt, sondern nur um solche, die einen körperteil, ein kleidungsstück, oder auch den teil eines leblosen dinges bezeichnen. Dass dem so ist, gebe ich gern zu, und ich hatte natürlich auch bemerkt, was ja auf den ersten blick einleuchtend ist, dass es sich hier um körperteile etc., oder besser um eine zugehörigkeit überhaupt (bolsivacio, pasicorto), handelt; allein es schien mir nicht nöthig, dies in die definition einzuführen».

${ }_{16}$ Mucho se ha escrito sobre la equivalencia de /de/ y /que/ como transpositores y subordinantes adjetivales, $c f$. últimamente Granvik (en prensa).

17 García Lozano (1993[1978]: 211) sostiene la opinión contraria: «De los tres modelos de interpretación que nos ofrecen los diccionarios, el tipo III) cariancho $\rightarrow$ ancho de cara es, en la mayoría de los casos, el más adecuado para interpretar el significado de los compuestos, por el hecho de ir acompañado el adjetivo de un complemento de limitación y posesión». Y ello porque "Desde el punto de vista sintáctico, el miembro determinante del conjunto es, indudablemente, el adjetivo y no el sustantivo, ya que el resultado de la fusión es un vocablo perteneciente a la clase de los adjetivos» (ibidem). Por supuesto, aquí el autor emplea el término determinante en el sentido de 'principal, más importante', no en el sentido en que suele emplearse en gramática $\left(c f .2 .^{\text {a }}\right.$ acepción del $\left.D L E\right)$ de que 'el adjetivo determina al sustantivo', lo cual implicaría que el sustantivo es el elemento principal y el adjetivo el secundario. 
miembros no puede explicarse como 'de burro(s) ciego(s)', 'que tiene (el/los) burro(s) ciego(s)' o 'ciego de burro', sino que lo subyace a esta construcción es la comparación 'ciego como un burro'. Y, en este caso especial (y raro, si atendemos a su frecuencia), el adjetivo es el elemento determinado y el sustantivo, el elemento determinante, lo mismo que ocurre, por ejemplo, en compuestos griegos del tipo $\mu \varepsilon \lambda \iota \eta \delta$ 's ('dulce como la miel') y alemanes del tipo honigsüß ('dulce como la miel') o schneeweiß ('blanco como la nieve') ${ }^{18}$. Debido a su rareza, el modelo burriciego siempre ha sido muy marginal, de modo que los neologismos que se crean siguen normalmente el modelo de pelirrojo: así, por ejemplo, carnigallináceo y pelipúntico ${ }^{19}$, que provienen de un sintagma "sustantivo + de + sustantivo" (carne de gallina, pelos de punta), cambian la categoría de su segundo miembro formando los adjetivos derivados gallináceo y púntico. E, incluso, en el caso de que sea una locución nominal comparativa el origen del nuevo compuesto, no se copia el modelo de burriciego ${ }^{20}$,

${ }^{18}$ Estos compuestos, llamados armstrong por antonomasia (Uhlich 1997: 21) o bahuvrīhi invertidos (Tribulato 2006:164), son poco frecuentes en las lenguas indoeuropeas: casi exclusivos de la antroponomástica en los grupos celta y, en menor medida, germánico. Constan, normalmente, de un primer miembro sustantivo que determina a un segundo miembro adjetivo. Los compuestos denominados bahuvrīhi o posesivos, de los que trataremos infra con más detenimiento, son adjetivos que muestran normalmente el esquema «adjetivo determinante + sustantivo determinado": un ejemplo típico es nuestro pelirrojo, el cual, sin embargo, presenta la novedad de un cambio en el orden de los constituyentes (sustantivo determinado + adjetivo determinante) de acuerdo con la evolución sintáctica de las lenguas románicas. Atendiendo a la relación entre sus miembros, de los que el segundo es siempre determinatum y el primero es siempre determinans, podemos distinguir varios subtipos, como, por ejemplo: 1 . El que da nombre al grupo, armstrong, que presenta el esquema morfológico de «sustantivo + adjetivo" y la relación semántica 'adjetivo de cualidad precisado (en su ámbito de aplicación) precisado por un sustantivo de parte del cuerpo'. 2. El que, con el mismo esquema morfológico, expresa una relación semántica de 'comparación' entre ambos miembros, siendo normalmente el primer miembro sustantivo el término de la comparación: son los $\mu \varepsilon \lambda \iota \eta \delta \eta \dot{s}$, honigsüß y schneeweiß citados más arriba. 3. El que, con el mismo esquema morfológico, expresa una 'relación semánticoreferencial específica' entre sus miembros: la que, en cada caso, se da entre ellos: oivoßapńs (lat. vino-

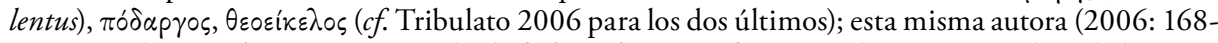
169) considera a $\pi \circ \delta \dot{\eta} v \varepsilon \mu \circ$ un ejemplo de bahuvrīhi invertido por ser el primer miembro el elemento regente, lo cual es rarísimo en las lenguas indoeuropeas antiguas: el bahuvrīhi normal es $\alpha \varepsilon \lambda \lambda \alpha^{\prime} \pi \circ \varsigma$. Es de destacar que este esquema morfológico concreto presenta entre ambos miembros una relación semántica que es siempre aquí la de 'comparación' ( $c f$. Tribulato 2006: 176, quien piensa que estos compuestos determinativos derivan de primitivos sintagmas con un adjetivo precisado por un sustantivo en acusativo [de relación]).

19 Sobre este compuesto y otros por el estilo, $c f$. la columna de Javier Marías (en El País del 16 de diciembre de 2018) titulada Palabras que me impiden seguir leyendo: «En cuanto al horroroso y mal formado "ojiplático", que ya ha pedido su ingreso en el Diccionario, qué quieren. Pretender que a partir de "se me quedaron los ojos como platos" se cree ese engendro, es como aspirar a que también se incluyan "carnigallináceo", "pelipúntico" y "peliescárpico" para designar cómo nos quedamos cuando nos emocionamos o nos llevamos un susto" (https:// elpais.com/elpais/2018/12/10/ eps/1544456326_569657.html?rel=str_articulo\#1554636270239). No nos interesa aquí discutir si, en realidad, esta locución nominal comparativa no es más que parte de una locución verbal que la engloba (poner/tener/quedarse los ojos como platos).

${ }^{20}$ Ello no impide que un poeta como Quevedo, cuya capacidad neológica no necesitamos destacar, cree adjetivos como bolsicuerdos, referidos a hombres 'mesurados en sus gastos', 'que no gus- 
sino el de pelirrojo: tal hacen, por ejemplo, ojiplático y peliescárpico, que parten de las locuciones comparativas ojos como platos y pelos como escarpias. En todos estos casos, el elemento determinado es el primer miembro sustantivo y el elemento determinante el segundo miembro adjetivo (derivado de un sustantivo): así, en ojiplático, ojo sigue siendo el elemento determinado (y nuclear) del compuesto, mientras que el segundo miembro, plático, es un adjetivo derivado a partir de plato. En cuanto a la relación semántica entre ambos miembros, se puede parafrasear como 'con los ojos como platos', 'con los pelos como escarpias'. Volveremos sobre estos compuestos en el apartado que dedicamos a la tipología.

\subsection{ENDOCENTRISMO, EXOCENTRISMO Y NÚCLEO}

La oposición endocentrismolexocentrismo se ha empleado poco como criterio clasificador de primer o segundo nivel en español. Habitualmente se reserva para el tercer y cuarto niveles, como han hecho Bustos (1986) y Val Álvaro (1999), partiendo de una interpretación semántico-referencial de esta oposición que trasciende lo estrictamente lingüístico para centrarse en la designación más que en el significado. Por ello constituye una excepción la clasificación de Moyna (2011), estudiosa que aplica este criterio en el primer nivel y no desde una perspectiva semánticodesignativa, sino desde el punto de vista categorial.

Los términos de endocéntrico y exocéntrico se relacionan directamente desde Bloomfield $(1933)^{21}$ con la noción de núcleo (ing. head) del compuesto bien desde un punto de vista categorial, bien desde un punto de vista semántico (Scalise / Fábregas / Forza 2009: 57, hablan incluso de "Three Types of Exocentricity»). En primer lugar, desde el punto de vista de la categoría del núcleo, un compuesto es exocéntrico si presenta una categoría distinta a la de su núcleo, como ocurre con el adjetivo pelirrojo, ya que, para muchos lingüistas (entre los que nos contamos) ${ }^{22}$, el núcleo es

tan de despilfarrar dinero', en el que el segundo miembro adjetivo está determinado por un primer miembro sustantivo. Pero, en esta exposición, no nos vamos a detener en excepciones de este tipo.

${ }^{21}$ En efecto, esto ha sido así sólo a partir de Bloomfield (1933): para una exposición del desarrollo gramaticográfico de los términos bahuvribi, posesivo, mudado y exocéntrico, $c f$. Noordegraaf (1989), Whitehead (2013), Tribulato (2015: $44-47$ y 53-55) y Batista / Mora / Pérez Vigaray (2019: 86-92), entre otros.

22 Por supuesto, hay lingüistas (generativistas, en su mayoría) que consideran a rojo el núcleo de pelirrojo, con lo que no se produciría un cambio de categoría, pues, en este caso, tanto el elemento nuclear como el compuesto son adjetivos. Sin embargo, tal punto de vista se contrapone a varias evidencias histórico-comparativas: así, por ejemplo, el cambio del orden de palabras del latín a las lenguas románicas ha impuesto que se pase de sintagmas con el esquema «determinante + determinado» a otros de orden inverso: «determinado + determinante». Y esto pasa tanto en los sintagmas de "sustantivo + sustantivo" como en los de «sustantivo + complemento" y "verbo + complemento». De este cambio en el orden de palabras participan también los compuestos románicos, que, frente a los latinos, presentan el orden «determinado + determinante», como vemos en manilargo frente a longimanus y en portaestandarte frente a signifer. Y, comparativamente, el inglés o el alemán, que han mantenido el orden normal indoeuropeo de «determinans + determinatum» en los sintagmas 
el sustantivo pelo y el elemento determinante el adjetivo rojo, con lo que se ha producido un cambio de categoría: [[pel-(o) $\left.]_{\underline{\underline{N}}}[\mathrm{roj}-]_{\mathrm{A}}-\mathrm{o} / \mathrm{a}\right]_{\underline{\underline{A}}}$. Y un compuesto es endocéntrico si conserva la categoría de su elemento nuclear, como ocurre, por ejemplo, con el sustantivo camposanto, que mantiene la categoría de su núcleo, que aquí nadie duda que es campo: [[campo $\left.]_{\underline{N}}[\text { santo }]_{\mathrm{A}}\right]_{\mathrm{N}}$. En segundo lugar, desde el punto de vista semántico, se dice que un compuesto es exocéntrico cuando por su significado no es un hipónimo de su núcleo. Así, pelirrojo es también exocéntrico desde el punto de vista semántico, pues un pelirrojo o una pelirroja no son 'un tipo de pelo' sino 'una persona'. En cambio, camposanto es endocéntrico porque un camposanto es un 'tipo o especie particular de campo'. Por esta razón, en general, los compuestos posesivos se han considerado exocéntricos y los compuestos determinativos, endocéntricos ${ }^{23}$.

Actualmente, esta oposición ha caído un poco en desuso, de manera que un estudioso tan conocido como Bauer (2017: 35 y 37, respectivamente), desencantado ante la falta de potencia explicativa de la exocentricidad, se permite incluso alguna broma sobre el concepto de head:

Discussion of headness in compounds has tended to be restricted to the distinctions between examples like windmill and examples like egghead. The first has been said to be headed, the second not to be. [...] According to this criterion, mill is the head of windmill (because windmill is a hyponym of mill), but head is not the head of egghead, because egghead is not a hyponym of head.

Por esta razón, una de las tendencias más recientes ha consistido en abandonar el criterio categorial y semántico de la exocentricidad en favor de un criterio cognitivista que introduce la metáfora y la metonimia, tal y como ha hecho Zacarías Ponce de León (2018: 194-195):

Desde mi punto de vista, la noción de compuesto metonímico es mucho más adecuada y explicativa que la noción de exocentricidad. Es más intuitiva, encuentra su motivación en un concepto básico de la cognición humana y puede sistematizarse.

Volveremos sobre la cuestión de la exocentricidad y la endocentricidad en el apartado dedicado a la tipología.

nominales, también lo han mantenido en los compuestos, de manera que encontramos este esquema incluso en los compuestos con un núcleo verbal (a pesar de que ambas lenguas son SVO, no SOV): esta es la razón de la diferencia en el orden de los miembros entre los compuestos ing. redhaired y al. roothaarig, de una parte, y esp. pelirrojo, de otra.

${ }^{23}$ Normalmente adjetivos los primeros y sustantivos los segundos. Ahora bien, como, en general, los compuestos determinativos han constituido un cajón de sastre donde los distintos tipos sólo compartían la endocentricidad, o sea, que el compuesto pertenecía a la misma categoría que su elemento nuclear, en los compuestos determinativos de dos adjetivos está claro que la categoría final del compuesto va a ser adjetiva, salvo eventuales casos de sustantivación. 


\section{NUESTRA PROPUESTA DE CLASIFICACIÓN}

Como hemos dicho, frente a la práctica totalidad de las clasificaciones más extendidas de los compuestos léxicos del español, que se montan sobre criterios categoriales (categoría del compuesto, en el primer nivel, y categoría de los constituyentes, en el segundo), nuestra clasificación se basa en las relaciones gramaticales existentes entre los miembros del compuesto. Seguimos, en este punto, la línea propuesta por Bisetto / Scalise (2005: 331):

[...] an adequate classification of compounds has to be done primarily and consistently on grammatical grounds. And exactly the grammatical relations between the constituents of a compound can allow a homogeneous grouping (at least on a first level) of compounds of different languages.

\subsection{COMPUESTOS PROPIOS O MORFOLÓGICOS Y COMPUESTOS IMPROPIOS O SINTÁC- TICOS}

Respecto a las relaciones existentes entre los constituyentes de un compuesto léxico distinguimos, inicialmente, entre las relaciones propiamente compositivas y las relaciones sintácticas: tal diferencia es la que opone en el primer nivel de nuestra clasificación los compuestos propios o morfológicos a los compuestos impropios o sintácticos. Hablamos de relaciones compositivas - y, consecuentemente, de compuestos propios o morfológicos- para referirnos a aquellas en las que los dos constituyentes del compuesto no "copian" las relaciones de la sintaxis libre u oracional, sino que siguen patrones o esquemas formativos que más bien las conculcan como muestra el cambio o "salto»" ${ }^{24}$ de categoría gramatical que experimentan estos compuestos respecto de su núcleo: compuestos como pelirrojo y trotaconventos son categorialmente adjetivos y en esto se diferencian de sus núcleos respectivos, el tema nominal pel(i)- y el tema verbal trot(a)-. Por el contrario, hablamos de relaciones sintácticas -y de compuestos impropios o sintácticos- para referirnos a aquellas en las que los dos miembros constituyentes del compuesto léxico se combinan siguiendo las mismas reglas de la sintaxis libre, lo que desde el punto de vista categorial supone que los compuestos sintácticos presentan siempre la misma categoría que su núcleo ${ }^{25}$. Así, palabras com-

${ }^{24}$ Algunos autores hablan de salto de categoría para referirse al «cambio de clase formal que afecta al término sustantivo determinado" (Benveniste: 1977; 161): $c f$., por ejemplo, Moyna (2011: 197): "In all the patterns presented so far, one of the two compound constituents can be identified as the head, from which the compound inherits its syntactic properties and often also its semantic specifications. However, it is also true that in most compound classes seen so far, a subset of tokens 'jump' grammatical category, exhibiting distributional properties incompatible with those of the head constituent. In those cases, the resulting compound is said to be exocentric».

${ }^{25}$ En los casos en los que, como veremos, la relación entre los dos constituyentes de un compuesto impropio o sintáctico no es de 'determinación' o 'subordinación' y no se puede hablar, 
puestas como bocamanga, camposanto o buenaventura son, en todos los casos, categorialmente sustantivos como sus núcleos: boca, campo y ventura, respectivamente. En este mismo sentido, hace casi siglo y medio ya había hablado Meunier (1872: 5 y 1875: IX-X y XI, respectivamente) de compuestos sintácticos y compuestos asintácticos u ordinarios:

II y a en grec deux sortes de composés : les syntactiques et les asyntactiques. J'appelle syntactiques ceux qui sont conformes aux lois de la syntaxe, et asyntactiques ceux qui n'y sont pas conformes ${ }^{26}$ (1872: 5).

J'appelle composés syntactiques ceux dont le premier membre est à un cas, au cas voulu par la syntaxe, en opposition avec les composés ordinaires, ceux dont le premier membre est un thème, c'est-à-dire le cas général. Il y a lieu de les diviser en composés syntactiques de coordination et en composés syntactiques de subordination, selon qu'ils sont avec le dernier membre dans un rapport d'égalité ou de dépendance (1875: IX-X).

$[\ldots]$

Je pars d'un principe unique: ces composés sont nés de phrases complètes, dont on a laissé de côté quelque chose, tantôt le sujet, tantôt le régime direct, tantôt le régime indirect en totalité ou en partie. Le problème consiste à retrouver la phrasemère (1875: XI).

Citamos el precedente de Meunier (1872 y 1875) ${ }^{27}$ siguiendo una corriente actual que nos parece muy adecuada y que consiste en recuperar textos lingüísticos interesantes del siglo XIX (Tribulato 2015). En esta misma línea, Lindner (2011: 7-8), que tiene en cuenta a varios autores decimonónicos, cita a Brugmann (1906, II, 1: 35), quien también utiliza dos términos distintos para hablar de compuestos: «Zusammensetzungen oder Komposita sind als Einheit apperzipierte Wortgruppen. Die Benennung wird auf zweierlei angewendet, was ich als Worteinung und als Univerbierung unterscheide». Sin embargo, la distinción de Brugmann entre unidad léxica (Worteinung) y univerbación (Univerbierung) no responde a criterios

por tanto, de núcleo, i.e., los conocidos como compuestos coordinativos del tipo carricoche o rojinegro, la categoría del compuesto es siempre la misma que la de sus dos miembros constituyentes.

${ }^{26} \mathrm{Y}$ en nota a pie de página aclara: «On a généralement coutume d'appeler juxtaposés les mots que j'appelle composés syntactiques, et d'appeler composés ceux que j'appelle composés asyntactiques. Mais, comme en disant composés syntactiques, composés asyntactiques, j'exprime toujours le genre commun (composés), et la différence spécifique (syntactiques, asyntactiques), cette manière de dire ne peut induire en erreur».

${ }^{27}$ A propósito de Meunier dice Coseriu (1978: 240): «Ahora bien, en los estudios y en las consideraciones sueltas sobre la formación de palabras, desde L. F. Meunier, Les composés syntactiques en grec, en latin, en français, en italien et en espagnol, Paris, 1874 (en parte, incluso desde F. Diez, Grammatik der romanischen Sprachen, II, Bonn, 1838), se ha dicho de este tipo [coupe-papier] casi todo lo imaginable». Creemos que aquí el sabio profesor rumano ha fundido en una (con una fecha de publicación «intermedia») las dos obras que conocemos de Meunier (1872 y 1875): $c f$. también Lindner (2011: 18). 
morfológicos, sino exclusivamente semánticos ${ }^{28}$, pues ambos procedimientos pueden ser monolexemáticos o polilexemáticos, con la diferencia de que en la Worteinung se produce una unidad de sentido nueva cuyo significado difiere de la mera suma del significado de los miembros componentes, mientras que la Univerbierung no produce esa nueva imagen conceptual unitaria o unidad de sentido (einheitliche Gesamtvorstellung) ${ }^{29}$ :

Von der Worteinung ist zu trennen die Univerbation. Oft haben zwei Wörter im Satz gewonheitsmässig Kontaktstellung erhalten, ohne dass, wie bei der Worteinung, damit eine besondere Bedeutungsvereinheitlichung durch Bedeutungsmodifikation verbunden wäre (Brugmann 1906, II, 1: 37).

Sin entrar en más disquisiciones, recordamos que se ha mantenido hasta hoy (Lindner 2011: 11-12 y 37-38) esta oposición entre verdaderos compuestos y las univerbaciones que resultan de yuxtaposiciones ${ }^{30}$.

Desde el punto de vista formal, un rasgo notable que sirve para distinguir a ambos tipos es que los compuestos propios o morfológicos contienen siempre un tema en el primer miembro y una palabra en el segundo, como observamos en los dos esquemas o patrones compositivos sincrónicamente productivos en español: pelirrojo $\left[[\text { pel- }]_{\mathrm{N}} \mathrm{i}[\text { rojo }]_{\mathrm{A}}-\mathrm{o} / \mathrm{a}\right]_{\mathrm{A}}$ y trotaconventos, $\left[[\text { trota }-]_{\mathrm{V}}[\text { conventos }]_{\mathrm{N}}\right]_{\mathrm{A} / \mathrm{N}(\mathrm{m} / \mathrm{f})}$. Precisamente, la presencia de temas es una característica que comparten estos compuestos con los derivados y muestra la formación de palabras como un componente de la gramática con reglas propias y exclusivas. A este respecto estamos de acuerdo con

${ }_{28}$ Para una panorámica de los enfoques sobre semántica de los compuestos (generativo, cognitivo, psicolingüístico, onomasiológico y pragmático), $c f$. Pius ten Hacken (2016) y, especialmente, su contribución al volumen (Hacken 2016: 211-232), en cuya primera página leemos: «In the domain of the semantics of compounding, the central question is that of semantic interpretation".

${ }^{29}$ Cf. Brugmann (1906, II, 1: 35-36): «Der Anfang und das Wesen der Worteinung besteht darin, dass die Bedeutung von Wörtern, die im Satz einen engeren syntaktischen Verband bilden, in der Art modifiziert wird, dass dieser Verband konventioneller Ausdruck für eine irgendwie einheitliche Gesamtvorstellung wird. Diese Vorstellung deckt sich nicht mehr genau mit dem Sinne, der sich aus der Zusanimenfügung der durch die einzelnen Worte bezeichneten Vorstellungen ergibt, es ist eine Bereicherung des Sinnes eingetreten, der eine Verengerung des Bedeutungsumfangs entspricht, oder die Verwendung ist eine metaphorische geworden. So treten im Nhd. z. B. weisskobl weisser kohl, weisserübe (md. wéisserübe), landesverrat, schwarzer adler als 'Komposita' auf, weil sie Namen für bestimmte Gewächse, für ein bestimmtes Delikt, für bestimmte Wirtshäuser geworden sind» (la cursiva en einheitliche Gesamtvorstellung es nuestra).

${ }^{30}$ Cf. Lindner (2011: 18-19): «Die Bezeichnung Juxtaposition (frz. Juxtaposition, ital. giustapposizione, span. yuxtaposición) wurde als neulateinische Nachbildung von gr. $\pi \alpha \rho \alpha ́ \theta \varepsilon \sigma \iota \varsigma$ von Darmesteter 1875: 3ff für die noch nicht gefestigte, syntagmatische „composition apparente“ im Gegensatz zur „composition proprement dite“ („apposition“) festgeschrieben. [...] Wie auch beim deutschen Pendant Zusammenrückung ist die Verwendung in der modernen Kompositaforschung uneinheitlich und teilweise sogar widersprüchlich. [...] Auch die (deutschen) Kopulativkomposita werden manchmal als Zusammenrückungen interpretiert. [...] Unter Zusammenbildung hingegen versteht man eine Ableitung auf der Basis eines Kompositums bzw. einer zugrundeliegenden Wortgruppe (...), als deutsches Korrelat zu $\pi \alpha \rho \alpha \sigma \dot{v} v \varepsilon \sigma \_\varsigma$ bzw. decompositum». 
Hernández / Marqueta (2015: 141) cuando, al referirse a los compuestos de verbo y nombre, afirman que «la comunidad entre composición y derivación queda reforzada por una interpretación del papel de la vocal temática que le asigna una tarea similar en ambos procedimientos». Frente a lo que sucede con los compuestos morfológicos o propios, los miembros de los compuestos impropios o sintácticos son siempre palabras enteras y nunca temas, con la excepción de unas pocas decenas de compuestos coordinativos que presentan una - $i$ - (discutible, como vimos, si copulativa o compositiva ${ }^{31}$ entre ambos miembros. Así mismo, correlacionando la oposición endocéntrico/exocéntrico con los conceptos de núcleo y categoría gramatical del compuesto, tenemos que los compuestos propios o morfológicos son exocéntricos, mientras que los impropios o sintácticos son endocéntricos.

A modo de resumen, podemos decir que los compuestos propios o morfológicos son el resultado de la aplicación de los procedimientos productivos exclusivos de composición de palabras en español (al. Zusammensetzung), mientras que los compuestos impropios o sintácticos proceden del resultado de la aplicación de las reglas de la sintaxis libre y, por tanto, de la fijación de un sintagma en una sola palabra (ing. univerbation, al. Zusammenrückung) ${ }^{32}$.

\subsection{COMPUESTOS PROPIOS: NUCLEOVERBALES Y NUCLEONOMINALES}

En el segundo nivel de nuestra clasificación, dentro los compuestos propios o morfológicos y centrándonos en la categoría del núcleo, distinguimos los que presentan un tema verbal y los que no lo presentan. En los primeros, el tema verbal es siempre el núcleo, independientemente de la posición que ocupe ${ }^{33}$, razón por la cual

31 Es una discusión que remonta a Munthe (1889) y Baist (1899): a nuestro juicio (Pérez Vigaray / Batista 2002), se trata de una -i-copulativa (lo cual está claro en bastantes casos: vaivén, quitaipón, calicanto). Para Morera (1998) se trata de la - $i$ - compositiva. Lo cierto es que, a veces, la $-i$ - pertenece al primer término de estos compuestos coordinativos, como sucede en agridulce, donde, visto sincrónicamente, ha desaparecido la -o de agrio. Otras veces, se trata de antiguos genitivos latinos: santiamén, coliflor. La extensión de la -i-compositiva a partir del siglo xv debió de influir en la formación de los compuestos coordinativos, que, en catalán, se diferencian bien formalmente de los léxicos: all i oli / pèl-roig.

32 Cf. Bauer (2017: 51): «The difference between 'being made up of two or more lexemes' and 'being produced by a process of composition' is of importance in other places as well».

${ }_{33}$ El tema verbal suele ocupar la primera posición, salvo en los casos de arcaísmos (prolifico, causahabiente) o préstamos (vasodilatador). Para los compuestos latinos, Oniga (1988: 79-80) adopta una clasificación parecida a la que hemos propuesto aquí para los españoles: «La tipologia che proporremo cercherà dunque di tener conto di tutte le principali caratteristiche di un composto: la categoria lessicale del primo e del secondo membro; il rapporto sintattico tra i due membri; l'eventuale presenza di suffissi; il valore semantico dell'intero composto rispetto a quello dei suoi membri. Ciascun tipo di composto sarà dunque individuato da una particolare struttura morfologica complessa e da una particolare interpretazione semantica». Y, poco después, añade Oniga (1988: 81, en nota al pie): «Accogliamo dunque la distinzione fundamentale proposta da Dardano (1987, 147 ss.) fra "composti con base verbale" e "composti con base nominale"». 
los denominamos nucleoverbales: es el caso de lavaplatos, guardameta, misacantano, mortifero. En los segundos, los que no presentan un tema verbal, son, por supuesto, nucleonominales y el elemento nuclear es el sustantivo que ocupa la primera posición, como ocurre con pelirrojo.

\subsubsection{Compuestos nucleoverbales}

Estos compuestos constituyen el patrón más productivo en español. En su morfología aparece siempre un tema verbal + un nombre (normalmente, un sustantivo en plural o singular). Cuando se refieren a personas y animales, suele admitirse que son adjetivos, aunque la sustantivación siempre es posible y frecuente; cuando designan objetos, suele considerarse que producen sustantivos. Hay una relación de determinación entre sus dos miembros, de manera que el elemento verbal siempre es el elemento nuclear, determinado o regente, mientras que el sustantivo (o, en algunos pocos casos, un adjetivo sustantivado, como ocurre en matasanos, o en función adverbial como abrefácil, e incluso un adverbio) actúa como su complemento (o argumento). Presentan una morfología complicada, ya que, cuando son adjetivos claros (lameculos o mataperros) su género y su número no tiene nada que ver con el género y el número que presenta el segundo miembro, aunque es posible aprovechar sobre todo el número gramatical en los casos en que el compuesto se crea en singular (guardametalguardametas). En cuanto a su designación, se han utilizado siempre para caracterizar, la mayor parte de las veces jocosamente, a personas mediante la descripción de alguna de sus acciones típicas. En la mayoría de las ocasiones, estas creaciones son momentáneas y normalmente orales o propias de los medios de comunicación, por lo que no suelen perdurar pasando al diccionario. Pero, actualmente, suelen emplearse para designar objetos (máquinas, casi siempre), también caracterizándolos por su función típica (cortacésped, lavavajillas) y, normalmente, se consignan en el diccionario. Por último, a nuestro juicio y de algunos otros (pocos) estudiosos (Bork 1990, Hinojo 2003 y 2009), estos compuestos son la evolución natural románica de los compuestos latinos del tipo signifer ${ }^{34}$, si bien, como hemos visto, también hay arcaísmos (restos de compuestos latinos) y préstamos que presentan el elemento verbal en el segundo miembro.

El esquema más frecuente es el de [Tema Verbal $(-a / e)+\mathrm{N}] \rightarrow[\mathrm{N} / \mathrm{A}]$, que se aprecia en mataperros, abrelatas, guardameta, quitasol, etc. Pero también se documentan otros dos esquemas sincrónicos, mucho menos usuales: a) [TV-ale $+\mathrm{A}] \rightarrow$ $[\mathrm{N}, \mathrm{A}]$ matasanos, pisaverde, cantaclaro, abrefácil; b) $[\mathrm{TV}$-ale $+\mathrm{Adv}] \rightarrow[\mathrm{N}]$ bogavante, pasavante, catalejo, mandamás, saltatrás.

34 Aunque con dudas, porque los considera creación románica debida al cambio sintáctico, cf. Buenafuentes 2009: 224-225 y 237). 


\subsubsection{Compuestos nucleonominales}

Este segundo tipo de compuestos propios o morfológicos en español es de relativa frecuencia y productividad, pues, como se ha repetido con asiduidad, su primer miembro es un sustantivo que suele reducirse a la designación de partes del cuerpo humano o animal y, en menor medida, de piezas de ropa. Este primer miembro nuclear está determinado por un segundo miembro, en la mayoría de ocasiones un adjetivo, que precisa su ámbito denotativo. La categoría de estos compuestos es siempre adjetiva, aunque, por supuesto, son posibles las sustantivaciones, como ocurre, por ejemplo, con petirrojo. Si bien el esquema $[\mathrm{N}-i+\mathrm{A}] \rightarrow[\mathrm{A}]$ pelirroj-ola $[[\sqrt{\mathrm{pel}}(\mathrm{o})-$ $\left.\mathrm{i}]_{\mathrm{N}}+\left[{ }_{\mathrm{roj}}-\right]_{\mathrm{A}}-\mathrm{o} /-\mathrm{a}\right]_{\mathrm{A}}$ es el más frecuente y prototípico, también aparecen otras construcciones variantes que afectan siempre al segundo miembro del compuesto. Así, distinguimos cuatro variantes: a) en la primera de ellas, el segundo miembro del compuesto lo recubre un participio de pasado, dando lugar a compuestos del tipo alicaído $\left.\left[\left[V_{a l}(\mathrm{a})-\mathrm{i}\right]_{\mathrm{N}}+\left[\left[V_{\mathrm{ca}}-(\mathrm{e}) \mathrm{r}\right]_{\mathrm{V}} \text { íd- }\right]_{\mathrm{A}}-\mathrm{o} /-\mathrm{a}\right]_{\mathrm{A}} ; \mathrm{b}\right)$ la segunda variante está constituida por aquellos compuestos que presentan un participio de presente como segundo miembro determinante, originándose compuestos como caridoliente $[[\sqrt{ } \operatorname{car}(\mathrm{a})-\mathrm{i}]$ $\left.{ }_{\mathrm{N}}+\left[\left[{ }^{\text {dol }}-(\mathrm{e}) \mathrm{r}\right]_{\mathrm{V}} \text { nte }\right]_{\mathrm{A}}-\mathrm{o} /-\mathrm{a}\right]_{\mathrm{A}}$; c) la tercera variante contiene un adjetivo derivado como segundo constituyente del compuesto, dando lugar a formaciones del tipo de boquiconejuno $\left.\left[[\sqrt{ } \operatorname{boc}(\mathrm{a})-\mathrm{i}]_{\mathrm{N}}+\left[\left[\sqrt{ }_{\text {conejo }}\right]_{\mathrm{S}}-\mathrm{uno} / \mathrm{a}\right]_{\mathrm{A}}-\mathrm{o} / \mathrm{-a}\right]_{\mathrm{A}} ; \mathrm{d}\right)$ y, por último, la cuarta variante de este esquema compositivo la conforman aquellos escasos ejemplos en los que el segundo miembro de estos compuestos lo ocupa un sustantivo, como refleja el ejemplo cachicuerno $\left[\left[V_{\operatorname{cach}}(\mathrm{a})-\mathrm{i}\right]_{\mathrm{N}}+\left[{ }_{\text {cuern-o }}\right]_{\mathrm{S}}-\mathrm{o} / \mathrm{-a}\right]_{\mathrm{A}}$. Si, como sucede normalmente, el segundo miembro es un adjetivo o un participio, el compuesto suele presentar variación de género y número: pelirrojo, pelirroja, pelirrojos, pelirrojas ${ }^{35}$. Si el segundo miembro es un sustantivo, cosa que sucede rara vez, ocurre lo mismo que veíamos en los compuestos del tipo lavaplatos: el segundo miembro sustantivo sólo posibilita, en el significante del compuesto, la variación de número, de manera que estamos ante una situación parecida a la de ojiazul: palomo/paloma patipluma frente a palomas/palomos patiplumas.

En cuanto a la diacronía, parece evidente que, como ya quería Baist (1899), estos compuestos son la evolución natural románica de los compuestos latinos del tipo longimanus, si bien quedan en espańol compuestos de este tipo tomados directamente del latín y con el orden latino, como magnánimo o longevo. Trataremos a continuación de los compuestos sintácticos, los cuales no presentan mecanismos compositivos particulares, como los dos que hemos visto hasta ahora, sino que son construcciones fijadas procedentes de sintagmas de la sintaxis libre, como se ve claramente en hidalgo o pundonor, etc., que aún conservan la $-d$-del sintagma original ${ }^{36}$.

${ }^{35}$ Evidentemente, si el adjetivo final no presenta moción genérica, tampoco la presentará el compuesto, como se ve en ojiazul.

${ }^{36}$ Cf. Alarcos García (1955: 10): «Quevedo echa mano de la palabra hideputa que las gentes de su época usaban en la conversación, ya en son de agravio, ya en señal de elogio, o bien como exclamación sin otro valor que el interjectivo, y la somete a las modificaciones necesarias para 


\subsection{Compuestos impropios SUbORdinAtivos y COORDINATIVOS}

Por su parte, dentro del otro gran grupo de compuestos léxicos españoles, los compuestos impropios o sintácticos, en el segundo nivel de clasificación, y atendiendo a la relación sintáctica entre sus miembros constituyentes, distinguimos dos grandes subtipos: los compuestos sintácticos subordinativos (bocacalle, camposanto, buenaventura) y los compuestos sintácticos coordinativos (carricoche, rojinegro), siendo que, en estos últimos, no hay núcleo o, dicho de otra manera, hay dos núcleos. Como era de esperar, en los compuestos sintácticos coordinativos los dos miembros presentan la misma categoría, que, por supuesto, coincide con la del compuesto ${ }^{37}$.

\subsubsection{Compuestos subordinativos}

Clasificamos los compuestos sintácticos subordinativos atendiendo a criterios categoriales, de manera que los dividimos en sustantivos, que son aquellos en que, al menos, uno de sus miembros lo es, y adjetivos, en los que ninguno de sus miembros es sustantivo. En el caso de los compuestos sintácticos sustantivos de N+A / $\mathrm{A}+\mathrm{N}$ (aguardiente, nochebuena, altavoz, bajamar), el sustantivo es siempre el término determinado, ocupe la posición que ocupe; en cambio, cuando se trata de palabras compuestas de dos sustantivos (aguanieve, bocacalle, casatienda, pejerrey, varaseto), el determinado es siempre el primero. En el caso de los compuestos sintácticos subordinativos adjetivos, la construcción más normal es la de un adverbio que precede a un adjetivo deverbal (normalmente, un participio de presente o pasado), como vemos en los siguientes ejemplos: malcriado, malsano, biempensante, bienquerido.

\subsubsection{Compuestos coordinativos}

Se trata de un grupo de compuestos muy interesante, aunque reducido a unas pocas decenas de formaciones, en la mayoría de las cuales se detecta fácilmente la coordinación, representada por la conjunción coordinativa $y$, normalmente escrita - $i$-y asimilada, en muchos casos, a la - $i$ - compositiva de distinto origen (Pérez Vigaray / Batista 2002) $)^{38}$. Como era de esperar, los dos miembros de estos compuestos

acomodarla a la situación y condición de los que la utilizan. Así, los naturales de la isla de los arbitrios, discutiendo acerca del mejor procedimiento para enriquecer a su señor, "llamábanse hidearbitristas, como hideputas, contradiciéndose los arbitrios los unos a los otros y cada uno sólo aprobaba el suyo" (p. 236 a). Y, en el romance titulado Matraca de los paños y sedas, el bocací y el fustán "tratáronse de hideaforros / y hidetúnicas con pasos" (v. 372 a)».

37 Exceptuamos los compuestos coordinativos formados por dos verbos, que suelen dar un sustantivo, como vimos en los ejemplos de vaivén o quitaipón.

${ }^{38}$ Respecto de la - $i$ - compositiva latina, que es una vocal temática y está en el origen de la -i-compositiva del español (y de algunos compuestos italianos), escribe Bader (1962: 16-17): «Un 
suelen pertenecer a la misma clase de palabras y, a menudo, el resultado final suele expresar una suerte de "compromiso" entre la designación típica de ambos constituyentes: un ejemplo paradigmático es el baciyelmo que aparece en el Quijote, pero lo mismo sucede con muchos otros, como capisayo, sopicaldo, etc., entre los sustantivos, y con agridulce, rojinegro, etc., entre los adjetivos.

Atendiendo a la categoría final del compuesto, distinguimos, por una parte, los compuestos coordinativos sustantivos, formados de dos nombres entre los que media la conjunción copulativa (calicanto, carricoche) o, en mucha menor medida, de dos verbos y conjunción (vaivén, quitaipón, subibaja); y, por otra parte, los compuestos coordinativos adjetivos, formados por dos adjetivos unidos igualmente mediante la conjunción copulativa (rojinegro, anchicorto, rojiblanco). Por último, cabe señalar la existencia de un grupo de compuestos coordinativos en los que no se da la presencia de la conjunción copulativa y que se reduce prácticamente a adjetivos gentilicios, como, por ejemplo, francobelga, hispanofrancés, anglosajón, etc.

Nuestra clasificación se ve fortalecida, además, por el punto de vista diacrónico, pues refleja la evolución del sistema compositivo latino al español y a las restantes lenguas románicas en general. En efecto, aunque suelen destacarse más bien los aspectos innovadores ${ }^{39}$, como veremos con más detalle en el apartado siguiente, todo el componente de formación de palabras continuó sin apenas modificaciones del latín a las lenguas románicas, pues en latín también había compuestos morfológicos (longimanus y signifer) y compuestos sintácticos (res publica, terraemotus, aquaeductus), los primeros podían contener o no un elemento verbal y eran, generalmente, adjetivos, mientras que los segundos no solían contener elemento verbal y solían ser sustantivos.

thème en -i- est conservé au premier membre de münificus (...). Lorsque le premier membre est formé sur un nom en -i-, il est lui-même normalement en - $i$-. [...] Mais le - $i$ - final de premier membre de composé peut avoir une autre origine. [...] Car l'apophonie peut transformer en - $i$ - la voyelle finale du thème qui figure au premier membre de composé, à l'origine quand ce thème était un dissyllabe, type agri-(cola). [...] Mais la fréquence des premiers membres de composés dissyllabes terminés par un - $i$ - d'origine morphologique (müni-) ou phonétique (agri-) a étendu cet - $i$-à des termes où il ne se justifiait pas. C'est ainsi que des noms, qui ne sont pas des thèmes en -i-, présentent, par analogie, un - $i$-à la troisième syllabe (ahēnipess), à la quatrième (margarìtifer), ou au-delà (scytalosagittipelliger); - $i$ - finit par être senti comme caractéristique des premiers membres de composés. Et c'est lui qui sera employé comme "voyelle de liaison" quand l'adjonction d'un thème consonantique faisait difficulté (part-i-ceps). L’extensión analogique de -i-confère donc au premier membre de composé une grande uniformité, qui empêche de distinguer ce qui était thème en - $i$ - et thème en -o- (ou - $a-$-): docto-, aula- deviennent docti- (loquus), auli- (coqua)». Cf. también Oniga (1988: 69-76) y (1992: 99) y Bauer (2017: 82-93), que se detiene mucho en los distintos tipos.

39 Cf., por ejemplo, Sánchez (2009: 105): «En general, podemos decir que, salvo en los pocos casos que hay herencia directa del latín, los romances hubieron de reestructurar e innovar los esquemas de composición de palabras, desarrollando muchos patrones que eran incipientes en latín vulgar, hasta superar en riqueza y variedad al propio latín». 


\section{APUNTE DIACRÓNICO}

Por lo que respecta a la historia del español, Pérez Vigaray (2010 [1994]) ha defendido el estudio conjunto de los dos grandes tipos de compuestos propios o morfológicos españoles: pelirrojo y guardameta. Estamos convencidos de que ambos tipos no se pueden separar, pues su evolución desde el latín al castellano ha sido paralela. El origen latino de ambos tipos está claro: tenemos, por un lado, longimanus y, por otro, signifer, ambos adjetivos, en los que se aprecia el orden de palabras típico del latín con el elemento determinante precediendo al determinado, exactamente lo contrario de lo que sucede en las lenguas románicas, pero que, en los dos casos, obedece al orden sintáctico normal en los respectivos sistemas (Buenafuentes 2009 sigue este mismo planteamiento). Además, los correspondientes ejemplos bajolatinos, en este caso, barbirasus y poscinummia testimonian este cambio de orden de palabras, pues en ambos el determinatum precede al determinans, de manera que bajo forma latina revelan ya el orden románico. Pero no solamente eso, sino que también, como hemos dicho, en español quedan restos del sistema latino en ejemplos como longevo y misacantano, antiguos adjetivos sin duda pero con un orden distinto al del español actual.

Sabemos que, tradicionalmente, se ha considerado al tipo pelirrojo como adjetivo y al tipo guardameta como sustantivo, de la misma manera que suele hablarse de adjetivos en el caso del latín longimanus, pero de sustantivos en el caso de signifer o lucifer. Ahora bien, en latín está claro que todos esos compuestos son adjetivos: basta fijarse en construcciones como navis stultifera mortalium. El que signifer o lucifer se hayan lexicalizado para designar entes concretos no empece en absoluto su antiguo carácter adjetivo. En este sentido, de la misma manera que nadie discute el carácter adjetivo de pechirrojo, tampoco se discute la sustantivación operada en petirrojo o patialbillo, designaciones de un determinado tipo de pájaro y gato, respectivamente. Desde luego, no es una novedad que los adjetivos se puedan sustantivar: y eso es lo que ha ocurrido también en Lucifer, por ejemplo. Por la misma razón, creemos que también está fuera de duda el carácter adjetivo de una construcción como misacantano, sustantivada después para referirse al 'sacerdote que celebra su primera misa' y tomada como sustantivo masculino, en lo que sin duda ha influido el hecho de que, en la Iglesia católica, no hay sacerdotisas ${ }^{40}$. Por la misma razón designativa, el $D L E$ considera el adjetivo compuesto trotaconventos un sustantivo femenino con el significado de 'alcahueta, tercera, celestina ${ }^{\text {'41 }}$. Frente a esto un estudioso de la talla de Jens Lüdtke (2011: 403) afirma:

${ }^{40}$ La palabra sacerdos es un adjetivo compuesto en latín, cuyo primer miembro es el adjetivo sacer y el segundo un nomen agentis deverbal, de la raíz ${ }^{*} d h e H_{l}$, que encontramos en facio, y, en principio, servía tanto para masculino como para femenino: las formaciones sacerda, sacerdota y sacerdotissa son recientes (cf. Bader 1962: $\$ \$ 21,76$ y 135).

${ }^{41} \mathrm{Y}$, en otro orden de cosas, también están sustantivados compuestos verbonominales, que son formaciones, en origen, adjetivas, como abrelatas, rompecabezas y trabalenguas. Más difíciles de sustantivar resultan, sin embargo, engañabobos o sacaperras, si bien engañamuchachos (designa- 
Por la alta disponibilidad de los compuestos de verbo + complemento, sobre lo que los lingüistas que los han estudiado están de acuerdo, el empleo de diccionarios es particularmente inadecuado, porque se incluyen solamente los compuestos que se han vuelto usuales. A pesar de este inconveniente, es costumbre apoyarse sobre todo en los diccionarios. Este método no permite captar la fase fugaz de la creación de un compuesto de verbo + complemento en el discurso, lo cual podría ser clave para la comprensión de este tipo, ya que, según parece, estos compuestos se crean como adjetivos que determinan sustantivos y que con el tránsito al acervo lingüístico sufren una sustantivación por medio de una elipsis. La frecuencia con que aparece el masculino como la forma sin marcas en estos compuestos, apunta también a un nacimiento por la vía de la conversión.

En suma, ambas formaciones eran originariamente adjetivas (Tabares / Pérez Vigaray Batista 2012; Tabares / Batista 2013; Giammatteo / Trombetta 2015) $\mathrm{y}$, a nuestro juicio, este procedimiento formativo sigue creando adjetivos en español actual, si bien, evidentemente, son susceptibles de sustantivación y, en muchos casos, parece que los nomina instrumenti se crean ya desde el propio esquema compositivo como sustantivos. Esta es la postura de Bader (1962: 413) respecto a los compuestos latinos (la cursiva es nuestra) ${ }^{42}$ :

On ne saurait mieux en juger qu'en distinguant, parmi les composés, les composés dérivés des composés non dérivés, et les composés adjectifs des composés sustantifs. Seule, cette double distinction permet de comprendre l'histoire et le mécanisme de création des composés nominaux. Les plus anciens d'entre eux ont été à la fois des composés dérivés et des composés adjectifs. De ce caractère adjectif témoigne la formation de ces composés, où le degré radical le plus ancien est le degré zéro, et où les problèmes du genre grammatical liés à l'adjectivation d'un substantif jouent un rôle notable. De plus, la valeur adjective des composés est particulièrement sensible dans les bahuvrihi, qui transforment un substantif simple en adjectif au second membre; mais c'est aussi celle des noms d'agent: un nom d'agent n'est autre qu'un adjectif verbal, et, au fur et à mesure que les anciens noms d'agent se substantivent, on voit se développer de nouveaux composés, mieux caractérisés dans leur forme comme

ción de un pájaro en Fuerteventura) y tragaperras muestran sustantivación. Pero ¿por qué se asigna a trotaconventos y tragaperras el género femenino y a guardameta el masculino? Simplemente porque se piensa en el referente habitual de la Celestina y la máquina, por un lado, y en un equipo de hombres, por otro. Pero si los analizamos con un poco de detenimiento, llegamos a la conclusión de que se trata de adjetivos, que, como tales, cumplen todas las propiedades de los adjetivos, aunque, claro está, son adjetivos morfológicamente sui generis debido a su morfología particular. Y, en cuanto a la sustantivación, ambos tipos, como todos los adjetivos, pueden sustantivarse: lo observamos en petirrojo y portaaviones, por ejemplo; pero no hay que confundir la posibilidad de sustantivarse con el hecho de ser sustantivos.

${ }^{42}$ Lo mismo afirma Oniga (1992: 108): «To conclude, we can affirm that the most productive types of compounds in Latin, the synthetics [= compuestos núcleo-verbales, del tipo signifer, sacerdos] and bahuvrihi [tipo longimanus], ... in a certaine sense are all exocentric compounds, in that the 'centre' of the compounds comes to be 'outside' the two compositional members in the position occupied by the suffix». 
adjectifs (ainsi -ficus à côté de -fex). À cette époque, les «composés» substantifs ne pouvaient être que tirés de ces adjectifs (type iüdicium < iüdex).

Retomando, pues, algunas consideraciones que hemos expuesto a lo largo de este trabajo, podemos decir que el español, como el latín, repugna la composición (Meillet y Vendryes 19795: 420-421) y muestra dos tipos principales de compuestos morfológicos: uno sin elemento verbal (pelirrojo) y el otro con elemento verbal ( guardameta), que responden, en principio, a procedimientos para formar adjetivos ${ }^{43}$.

Por su parte, Bork (1990: 241-242) ofrece 39 ejemplos (Gather 2001: 202) de compuestos verbonominales latinos con el orden románico de $\mathrm{V}+\mathrm{N}$ desde el siglo III a.C. hasta el vi d.C. Y tanto Bork (1990: 382-383) como Hinojo Andrés (2003 y 2009) piensan que siempre hubo compuestos verbonominales en latín con el orden citado y que tales compuestos proliferaron en las lenguas románicas siguiendo el orden normal de las palabras en la oración. En este sentido, Bork (1990), como Coseriu (1978: 239-240), considera que los compuestos verbonominales románicos son herencia latina y que deben su preponderancia a la influencia griega:

Es erscheint möglich, die spätlateinisch-romanische VE-Komposition als Erben des untergehenden EV-Musters anzusehen (Bork 1990: 386).

[...]

Entgegen der herrschenden Meinung, nach der das VE-Bildungsmuster eine Neuerung der romanischen Sprachen sein soll (oben Kapitel 2), weist diese Arbeit eine lückenlose ... Tradition vom Indogermanischen über das Griechische und das (Vulgär)Lateinische bis zum vor- und frühromanischen Mittelalter nach. ... Damit ist diese Arbeit zugleich ein Kapitel in dem umfangreichen ... Buch über den prägenden Einfluß des Griechischen auf die Struktur des Vulgärlateins und damit der romanischen Sprachen (Bork 1990: 387).

43 Considerar adjetivos a estos compuestos nos evita, además, el problema de forzar la categoría de su cabeza o head, tal y como ha hecho Soledad Varela (1990), postura discutida por Jiménez Ríos (1999). En efecto, algunos estudiosos, convencidos de que la categoría del determinatum es la misma que la categoría final del compuesto, aducen que, en pelirrojo, el núcleo es rojo y que no se produce ningún cambio de categoría. Exactamente lo mismo ocurre en los compuestos verbonominales, en los que también se opera un salto de la categoría del elemento determinado, verbo, que pasa a la categoría adjetiva (o sustantiva) del compuesto. Ambas formaciones resultan, pues, también paralelas desde este punto de vista y se evitan las interpretaciones distorsionadas que ven el núcleo de pelirrojo en rojo y el de abrecartas en un supuesto primer miembro sustantivo agentivo, abre, con sufijación ø. La -e-de abre- en abrecartas no es más que la vocal temática del verbo abrir, aprovechada como nexo de unión entre ambos miembros. Exactamente de forma paralela a como se aprovecha la - $i$ - de unión entre pel-y rojo en pelirrojo. Por todo ello creemos que los compuestos verbonominales españoles se dejan analizar mejor como adjetivos que como sustantivos. De forma muy parecida, y tras realizar todas las pruebas que se suelen requerir para distinguir adjetivos de sustantivos, se han expresado Giammatteo / Trombetta (2015: 206) sobre compuestos como quitapenas, abrelatas y rompecorazones: "Consideramos, por tanto, que los ejemplos y argumentos esgrimidos permiten postular el origen adjetival de los compuestos estudiados». 
Sin embargo, esta posición, que, en general, compartimos ${ }^{44}$, no convence a Gather (2001: 204 y 206, respectivamente), quien, volviendo al planteamiento tradicional, considera que los compuestos verbonominales románicos son una innovación con respecto al latín:

In Übereinstimmung mit der romanistischen (und lateinischen) Tradition gehe ich - contra Bork (1990)- davon aus, daß es sich bei den VNK um eine Neuerung des Romanischen gegenüber dem Lateinischen handelt.

[...]

In den romanischen Sprachen, oder besser: im Protoromanischen, entsteht also durchaus eine neue Struktur, gleichsam wie Phönix aus der Asche. Der Untergang des alten Kompositionsprinzips und die Entstehung und Konsolidierung des neuen Typs stellen eine radikale Umstrukturierung der Kompositionsmorphologie dar.

En este sentido, Gather (2001: 207-208) admite la influencia del cambio sintáctico del orden de palabras en los compuestos verbonominales románicos, pero contraargumenta que el cambio de SOV a SVO, que también ha ocurrido, por ejemplo, en inglés y griego moderno, no ha conllevado que el miembro determinado de los compuestos ingleses o del griego moderno vaya delante del miembro determinante, sino que, en estos compuestos, el elemento nuclear sigue siendo normalmente el segundo y está, por tanto, a la derecha (right-headed compound system; cf. Tribulato 2015: 45-46). A esta objeción de Gather hay que responder lo siguiente: el hecho de que, en inglés y griego moderno, la mayoría de los compuestos sigan manteniendo el núcleo a la derecha está relacionado con que, en estas lenguas, como en la mayoría de las lenguas indoeuropeas modernas, se ha producido, en general, el cambio de orden SOV a SVO, pero esto no ha ocurrido en los sintagmas nominales, donde el elemento determinante sigue precediendo al determinado $(A+N$; $\mathrm{CN}+\mathrm{N})$, mientras que, en español y en las demás lenguas románicas, el cambio de orden SOV a SVO ha venido acompañado del cambio en el orden tradicional indoeuropeo de los sintagmas nominales, de manera que lo normal es $\mathrm{N}+\mathrm{A}$ y $\mathrm{N}+$ $\mathrm{CN}$, con lo que es de esperar que el elemento nuclear esté siempre a la izquierda ${ }^{45}$.

${ }^{44}$ Aunque nos parecen sugerentes las ideas de Coseriu (1977: 152-170, 231-280) acerca de la influencia griega sobre el latín vulgar y las lenguas románicas, se nos antoja muy remota la posibilidad de una influencia griega en estos compuestos románicos.

${ }^{4}$ Por supuesto, estamos hablando en general, ya que, por ejemplo, en alemán, el orden SOV se mantiene en las oraciones subordinadas. Y, en español, ejemplos como burriciego tienen el núcleo a la derecha. Pero es que, en este caso, como hemos dicho, se trata de compuestos procedentes de sintagmas fijados (ciego como un burro), lo cual sucede también, por ejemplo, en griego anti-

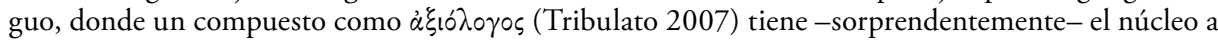
la izquierda por proceder también de un sintagma fijado. 


\section{NOTAS TIPOLÓGICO-CONTRASTIVAS}

Todas las siguientes consideraciones se enmarcan en la amplia e intrincada cuestión de la exocentricidad, denominación surgida en el ámbito de la lingüística indoeuropea a finales del $\operatorname{XIX}^{46} \mathrm{y}$, en general, relacionada con el término indio bahuvrīhi $i^{47}$. En cuanto a la cuestión de su origen, no nos parece que tenga razón Risch (1974: 182-183) y que los compuestos posesivos deriven de compuestos determinativos ${ }^{48}$, tal y como pensaba Brugmann (1905-06: 60-61), para quien los compuestos originarios son determinativos (para entendernos, proceden de sintagmas, como nuestros hidalgo, zarzamora, camposanto y bajamar), mientras que los bahuvrìhi constituyen una excepción porque predican una cualidad esencial de un sustantivo externo a ellos, por lo que le parece bien el término de Aleksandrow (1888: 110) ${ }^{49}$, esocentrische / exocentrische Zusammensetzung) y los llama exocéntricos porque «das Subjekt nicht in dem Kompositum, sondern außer ihm liegend».

46 El término data de 1888 y se debe a Aleksandrow (1888) (cf., entre otros, Noordegraaf 1989 y Whitehead 2013). Partiendo del concepto de bahuvrīhi de Pāninini (s. IV a.C.), referido a adjetivos, Bopp (1827 y 1852) desarrolló el concepto de compuestos posesivos y Aleksandrow (1888) denominó exocéntricos a compuestos adjetivos en la línea de Pāṇini, denominación que acogieron los neogramáticos alemanes. Sin embargo, desde Bloomfield (1933) se califica de exocéntricos a sustantivos compuestos e, incluso, sintagmas nominales, como vemos en whitecap y swallow-tail, respectivamente.

47 Benveniste (1977: 157) escribe al respecto: «Son los compuestos llamados bahuvrīhi, tipo de gran generalidad, del cual he aquí algunos representantes: inglés blue-eyed (azul+ojo) "ojiazul"; gr. kuno-képhalos (perro+cabeza) "(mono) con cabeza de perro": lat. quadru-pes (cuatro+pie) "(animal) de cuatro pies"; [...] La definición de estos compuestos siempre ha sido dificultosa, aunque haya acuerdo en el análisis empírico. Han recibido varias denominaciones. La de bahuvrīhi, la más inocente, designa en sánscrito la clase mediante uno de sus representantes. También se emplea "compuesto exocéntrico" para decir "cuyo centro cae fuera del compuesto", lo cual tiene el inconveniente de recurrir a una geometría azarosa (¿cómo va a estar el centro de un objeto fuera de él?), sin con ello elucidar la relación, que nada más es enviada fuera del compuesto. Más claro, al menos en su sentido inmediato, es el término "compuesto posesivo", y contiene, como se verá, una pizca de verdad, pero no deja de ser aproximativo y mal definido, y en suma inadecuado».

${ }_{48}$ A esta dirección apunta el hecho histórico de que los compuestos del tipo pelorrojo o barbanegra precedan a pelirrojo o barbinegro, que se desarrollan solo a partir del siglo xv (Munthe 1889 y Moyna 2011). En este sentido, Montes Giraldo (1977: 653) escribe: «los tipos de compuestos sust. + adj. que se dan en Colombia son sust. + adj. con el primer elemento terminado en -i (aliabiertos, bigotipintao, boquiabierto, etc.), que llamaré tipo A, y sust. + adj. sin modificación del primer elemento, que nombraré tipo B». Y, en nota a pie de página, explica: «El tipo B representa un estadio anterior, no sólo históricamente (véase adelante), sino también estructuralmente en cuanto es evidentemente una composición menos auténtica, ya que, aunque no mantenga la variabilidad morfológica de sus dos componentes, sí mantiene la concordancia entre éstos (al menos en los compuestos recogidos en Colombia: cabezadura, huevoduro, cachobroco) y no, por consiguiente, con el sustantivo a que se aplica».

49 En concreto: «1. Zusammensetzungen mit dem Schwerpunkte der Bedeutung in einem der Bildungselemente (esocentrische). 2. Zusammensetzungen ohne Schwerpunkt der Bedeutung in den Bildungselementen (exocentrische): der Schwerpunkt liegt ausserhalb derselben, in dem Worte, auf welches sich die Zusammensetzung als Adjectivum bezieht». 
De hecho, Brugmann (1905-06: 68) piensa que compuestos exocéntricos

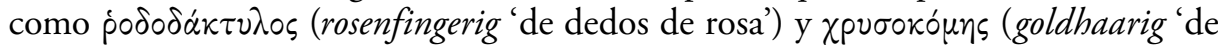
cabello dorado'), que entiende y traduce como adjetivos, fueron sustantivos con que se daba nombre a seres caracterizados por tener dedos rosáceos y pelo dorado, respectivamente ${ }^{50}$. Y esta concepción, pasando por Bloomfield (1933), ha llegado a Scalise / Fábregas / Forza (2009) y Bauer (2017: 64-71).

Comparando los compuestos del griego antiguo con los del alemán moderno, Risch (1981[1944]: 3-4) constata que, a pesar de ser muy numerosos en ambas lenguas, son de tipo muy diferente, pues, frente a lo que ocurre en griego, donde los compuestos suelen ser de rección verbal o posesivos, la gran mayoría de los compuestos alemanes son determinativos, en los que el primer miembro (Bestimmungswort) determina o precisa al segundo, que es el núcleo (Grundwort) del compuesto ${ }^{51}$, de manera que tales compuestos son, en realidad, hipónimos del sustantivo o adjetivo que les sirve de base: así, los compuestos del sustantivo Pferd (caballo) o del adjetivo rot (rojo) precisan subclases de caballos (sustantivos) o de rojos (adjetivos): Reitpferd (caballo de monta), Rennpferd (caballo de carrera), Zugpferd (caballo de tiro), Wil$d p f e r d$ (caballo salvaje), etc.; y rosarot (rojo rosáceo), hellrot (rojo claro), dunkelrot (rojo oscuro), ziegelrot (rojo teja). Muy distinto nos presenta Risch (1981[1944]: 5) el panorama de los compuestos griegos, de los que nos ofrece los siguientes datos (traducimos directamente al español y ponemos en nota el texto original $)^{52}$ :

Un cuadro muy diferente ofrecen los compuestos nominales griegos. Aquí los compuestos determinativos son llamativamente escasos; tienen mucha mayor importancia los compuestos de rección verbal (en el sentido más amplio), que constituyen un $60 \%$ de todos los compuestos nominales, y los compuestos posesivos (entre un $25 \%$ y un 30\%). [...] El resto se reparte entre los compuestos de rección

${ }^{50}$ Literalmente: «Er [der mangelhafte Geschlechtsausdruck] beruht allerdings auf dem substantivischen Ursprung dieser Komposita. Aber es hat keine Umwandlung eines Determinati-

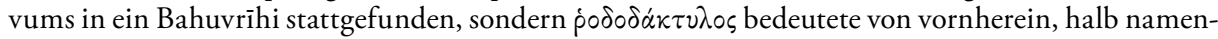

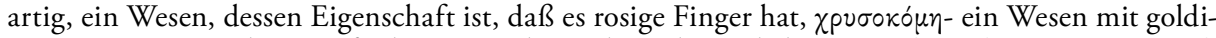
gem Haar». Sin embargo, $c f$., al respecto, las réplicas de Neckel (1906: 249-254) y Petersen (1914: 254-285), entre otros. Por su parte, también Buck (1933: 355) afirma que los compuestos posesivos eran originariamente determinativos que, a través de su uso como epítetos en aposición a otros nombres, han llegado a adquirir el valor adjetival que presentan.

${ }^{51} \mathrm{Y}$ en nota explica que se refiere aquí a los compuestos que mantienen el carácter sustantivo del segundo término, es decir, que son hipónimos de este. Por esta razón cita las denominaciones respectivas de Debrunner («nicht mutierte Determinativkomposita») y Schwyzer («endozentrische Determinativkomposita»).

52 En sus palabras: «Ein ganz anderes Bild ergibt die Gruppierung der griechischen Nominalkomposita. Hier sind ausgerechnet die Determinativkomposita auffallend selten, es überwiegen die verbalen Rektionskomposita (im weitesten Sinn), die etwa 60\% aller Nominalkomposita ausmachen, und die Possessivkomposita (Bahuvrīhi), zu denen 25-30\% der Komposita gehören. Der Rest verteilt sich fast ganz auf die präpositionalen Rektionskomposita (Hypostasen) und ähnliche Bildungen, ferner auf die adjektivischen und substantivischen Determinativkomposita. Dabei zeigen die Stichproben keinen wesentlichen Unterschiede zwischen der Texte verschiedener Zeitepochen und verschiedener Literaturgattungen». 
preposicional (hipostáticos) y construcciones semejantes, además de los compuestos determinativos sustantivos y adjetivos. Y las muestras tomadas de textos de diferentes épocas y géneros literarios no reflejan diferencias esenciales.

Ahora bien, ¿qué se entiende por determinativo en el sintagma compuesto determinativo (al. Determinativkompositum) ${ }^{53}$ ? Pues, simplemente, un hipónimo «concreto» del miembro determinado. Así, en alemán, Arbeitszimmer (cuarto / habitación de trabajo), Wohnzimmer (sala de estar), Schlafzimmer (dormitorio), Kinderzimmer (cuarto/habitación de los hijos/niños), así como todos los compuestos que tengan como segundo miembro, que es el regente o determinado, al sustantivo Zimmer (cuarto/habitación) son hipónimos de Zimmer (cuartos o habitaciones) y, por tanto, son todos sustantivos del mismo género que Zimmer, que es neutro ${ }^{54}$. Y, ciertamente, la mayoría de los sustantivos compuestos alemanes (y muchísimos del griego moderno) son compuestos determinativos formados sobre el modelo de sustantivo + sustantivo, aunque también los haya, por supuesto, formados sobre el esquema adjetivo + sustantivo, del tipo de Dummkopf (tontaina, lit. cabeza tonta), Dickkopf (cabezota, lit. cabeza gorda), donde el elemento regente o determinado sigue siendo el que ocupa el segundo lugar y, por tanto, el sustantivo, con lo que el compuesto toma su misma categoría verbal y su género. En este segundo caso, aunque el resultado son sustantivos, como Rotkehlchen, Rotkäpchen, Rotbart, Rothaut, etc., suelen emplearse para caracterizar a personas y, eventualmente, animales, lo cual ha motivado que los estudiosos resalten su uso calificativo y los consideren «compuestos posesivos", como hacía Risch. Estos compuestos determinativos corresponden a nuestros compuestos sintácticos y, por supuesto, al igual que ocurría en español, también en alemán hay compuestos determinativos adjetivos, distinguiéndose dos tipos: a) los compuestos de adjetivo + adjetivo, de los que el determinado y regente es el segundo, porque así es el orden de palabras originario en las lenguas indoeuropeas y sigue siéndolo en la mayor parte de ellas, salvo en las románicas, de manera que schwarzweiß es nuestro 'blanco y negro', y, evidentemente, la categoría verbal del compuesto resultante es un adjetivo (como su segundo miembro); y b) los compuestos de sustantivo + adjetivo, mucho más interesantes, ya que un primer miembro sustantivo determina a un segundo miembro adjetivo, que es el regente por ocupar precisamente el segundo lugar, con lo que el compuesto resultante es un adjetivo: mann(es)hoch es 'del alto de un hombre', 'del alto de un hombre [normal]', 'que tiene la altura [media] de una persona', honigsü $\beta$ 'dulce como la miel', 'meloso' (cf. hochragend = ranghoch 'que tiene un alto rango' = 'alto de rango'). Esto es, mutatis mutandis, lo que ocurría con nuestro tipo burriciego.

53 Por cierto, el propio término Determinativkompositum es un compuesto determinativo, al igual que lo son las demás denominaciones de compuesto en alemán: Possessivkompositum, Verbalrektionskompositum, etc.

54 Curiosa es la metonimia que se opera en Frauenzimmer, caso en el que no nos podemos detener ahora. 
Sin embargo, frente a esta caracterización tradicional, en los últimos años, se ha puesto en tela de juicio la oposición entre compuestos endocéntricos (el caso más típico es el de los determinativos) y exocéntricos (el caso más típico es el de los posesivos o bahuvrì $i^{55}$ ). De hecho, en sus estudios sobre el griego moderno ${ }^{56}$, Ralli / Andreou (2012) han rechazado este concepto de exocentricidad tan generalizado como singular y han vuelto a la idea original de que los compuestos exocéntricos son en su mayoría adjetivos parasintéticos: según estos autores, compuestos neogriegos como $\chi \alpha \sigma o \mu \varepsilon ́ p \eta s$ (lit. 'pierdedías', 'que pierde el tiempo'), $\mu \alpha \kappa p \nu \mu \alpha \dot{\lambda} \lambda \eta \bar{s}$ ('pelilargo', 'de pelo largo') y $\kappa \alpha \lambda o ́ k \alpha \rho \delta o s$ ('de buen corazón') son compuestos adjetivos y exocéntricos, que siguen claramente modelos que remontan a Homero. En suma, Andreou / Ralli (2015: 183) concluyen que los compuestos del tipo equivalente al esp. lengualarga son sustantivos endocéntricos ('una lengua larga') que se pueden interpretar metonímicamente para referirse a una persona, mientras que los compuestos del tipo lengüilargo son adjetivos exocéntricos:

A Greek bahuvrihi compound, that is, a compound with the meaning 'having/ to have X', can be built on the basis of two formally distinct types. The first type is exemplified by voidokilia' 'ox-belly, met. having a big belly' which is a nominal endocentric compound which can be interpreted metonymically to denote 'one with a big belly'. The second type involves both compounding and derivation, in this particular order, and is exemplified by both the adjectival aspraloyátos 'having a white horse' and the verbal exocentric bahuvrihi kakonixtizo 'to have a bad night'57.

55 Cf. Andreou / Ralli (2015: 165): «We will assume that we should not collapse the two categories, that is, bahuvrihis and exocentric compounds despite that this diverges from the original proposal by Pāninini». Por lo tanto, Andreou / Ralli (2015: 166) distinguen los términos de bahuvrihi y exocéntrico, pues opinan que también hay bahuvrihis endocéntricos: «the distinction between the two main classes of Greek compounds and whether the second member is a stem or a word, can be used as a criterion for the identification of endocentric and exocentric bahuvribis» (la cursiva es nuestra). Pero, inmediatamente, añaden: «Let us first consider adjectival bahuvrihis since the bulk of Greek bahuvrihis belongs to this category». Así, a pesar de que estos autores encuentran bahuvrihis adjetivos, sustantivos y verbos, no dejan de destacar que los más importantes por su número y varie-

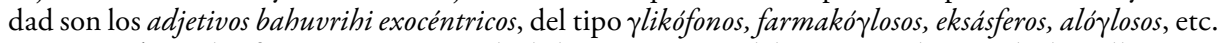

${ }^{56}$ La clasificación más aceptada de los compuestos del griego moderno es la de Ralli (2013: 30-36), quien, siguiendo la tripartición moderna, distingue sustantivos, adjetivos y verbos compuestos, siendo las siguientes las principales categorías: 1) Sustantivos: a) N + N: alatopiper (o) 'sal-pimienta' < alát(i) + pipér(i); b) A + N: stenosókak(o) callejuela estrecha < sten(ó) + sokák(i); 2) Adjetivos: a) A + A: asprokókin(os) 'rojiblanco' (lit. blancorrojo) < áspr(os) + kókin(os); b) N + A: iliokamén(os) 'quemado por el sol' < íli(os) + kamén(os); c) Adv + A: kakodimén(os) 'malvestido' < kak(á) + dimén(os); y 3) Verbos: a) V + V: anighoklín(o) 'abro-cierro' < anígh(o) + klín(o); b) N + V: xartopéz(o) 'juego [a las] cartas' < xart(iá) + péz(o); c) Adv + V: $\operatorname{arghopethén(o)~'muero~lentamente'~<~argh(á)~+~pethén(o).~}$

57 Andreou / Ralli (2015: 182-183) ejemplifican los bahuvrihis verbales con kak-o-nixtiz-o 'to have X', en este caso, 'tener (pasar) una mala noche', precisando que, frente a los bahuvrihi sustantivos, que muestran una palabra (word) en el segundo miembro, los bahuvrihi adjetivos y verbales muestran un tema (stem) al que se le añaden sufijos, por lo que combinan composición y derivación. En el caso de estos compuestos verbales, no cabe duda de que se trata de compuestos derivados de sintagmas, cosa que se ve perfectamente en $\kappa \alpha \lambda \eta \nu v \chi \tau i \zeta \omega\left(<\kappa \alpha \lambda \dot{\eta} v v_{\chi} \chi \tau\right)$. En este sentido, 
Por esta razón, Andreou (2014: 228-229) se basa en la categoría final del compuesto para distinguir entre los sustantivos y los adjetivos exocéntricos griegos vromóglossa y vromóglossos, que corresponden respectivamente a lengualarga y lengüilargo, y expone claramente las diferencias entre ambas construcciones, diferencias extrapolables al citado ejemplo español ${ }^{58}$.

En suma, nos parece incontestable la siguiente afirmación de Ralli / Andreou (2015: 165):

A fundamental difference, however, between the English red-head and possessive compounds in Greek and Sanskrit is that the latter function as adjectives and not as nouns. In other words, exocentricity in Greek and Sanskrit is morphologically marked and it is not a semantically defined phenomenon only ${ }^{59}$.

También añade Andreou (2014: 229-230) que las lenguas germánicas no suelen emplear bahuvrīhi adjetivos, sino que se limitan a usar metonímicamente sustantivos del tipo rostro pálido:

Although in most IE languages, adjectival bahuvrihis are more basic than nominal ones, Germanic languages followed a different route. Kastovsky (2009) argues that contrary to other IE languages, Germanic languages developed a very productive nominal bahuvrihi compounding system of the paleface type and also states that the adjectival pattern is manifested by only a handful of formations such as barefoot. In addition, he shows that adjectival bahuvrihis have been replaced by the so-called extended bahuvrihis which involve suffixation; hunchbacked $<$ bunchback ${ }^{60}$.

todos los compuestos verbales por incorporación (Pompei 2006) son parasintéticos, o sea, derivados de compuestos (o sintagmas) nominales.

${ }^{58}$ En efecto, extrapolando al español las citadas ideas de Andreou / Ralli (2015) tenemos que lengualarga es un compuesto sustantivo, endocéntrico o exocéntrico según aluda bien a una lengua larga, bien metonímicamente a alguien que habla mucho (y no bien de los demás); en cambio, lengüilargo sólo es un adjetivo exocéntrico, cuyo empleo coincide con el empleo exocéntrico de lengualarga. Pero lo que está claro es que ambos compuestos constituyen dos categorías morfológicas diferentes. Y es que, aunque, como hemos visto, no son decisivas (pelirrojolburriciego), las marcas formales son importantísimas: así, Andreou (2014: 265) afirma: "A comparison between the patterns [stem/word word] and [stem/word stem] shows that only compounds which belong to the latter pattern can be classified as exocentric. A Greek compound the second member of which is a word is always considered endocentric. This follows from that Greek exocentric compounds involve the combination of two stems (or a word and stem) which undergo derivation».

59 Así, atendiendo a la morfología y llevando las conclusiones de Andreou / Ralli (2015) hasta sus últimas consecuencias, tenemos que los compuestos exocéntricos y bahuvrīhi son siempre morfológicamente adjetivos, mientras que los sustantivos son morfológicamente compuestos endocéntricos, si bien susceptibles de interpretación exocéntrica por metáfora o por metonimia. $Y$, en cuanto a los bahuvrihi verbales, como reconocen nuestros autores, son parasintéticos, esto es, derivados de compuestos: los únicos compuestos verbales propiamente dichos, típicos sólo del griego

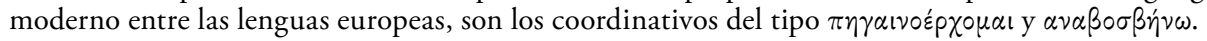

${ }^{60} C f$. asimismo Andreou (2014: 231): «In the previous chapter I argued that nominal bahuvrihis should be analyzed as endocentric compounds which are interpreted metonymically, whereas adjectival bahuvrihis should be accounted for by derivation (overt or zero). This means that a com- 
Sin embargo, aunque la mayoría de los adjetivos compuestos exocéntricos del griego moderno están formados por un adjetivo en el primer miembro y un sustantivo en el segundo, también los hay con elemento verbal ( $\chi \alpha \dot{\alpha} \omega \omega$ 'perder') en el primer miembro y un sustantivo en el segundo ( $\mu \varepsilon ́ p \alpha$ 'día'), como $\chi \alpha \sigma o \mu \varepsilon ́ p \eta s$ (Ralli / Andreou 2012: 72), que es un compuesto del mismo tipo que el español aguafiestas, con lo cual se desdibuja la oposición tradicional entre compuestos posesivos y compuestos de rección verbal en favor de la categoría final del compuesto, que es adjetivo en todos los casos citados ${ }^{61}$. Así lo resume Andreou (2015: 293):

The identification of a relation between exocentricity and change in the lexical category of the compound, i.e. a change from noun to adjective, is very important for our discussion on the distinction between endocentric and exocentric compounds (...) Debrunner also argues that the exocentricity of this compound manifests itself in the change of lexical category; the whole is an adjective despite the fact that both of its constituents are nouns. ... This means that exocentricity in languages such as Sanskrit and Greek is not just a semantic phenomenon since it is marked by formal means as well (e.g. change of category by affixation).

Resumiendo, la antigua distinción entre compuestos endocéntricos y exocéntricos, que se había "desmandado» a partir de Bloomfield (1933), empieza a recoger velas y parece que últimamente ha dejado de ser útil: así, afirma Andreou (2014: 264) que "Exocentricity is an epiphenomenon». De hecho, Bauer (2017: 71) concluye que «exocentricity can, where necessary, be explained in terms of figurative readings», conclusión a la que ya había llegado diez años antes en una aproximación tipológica a la composición:

Thus we can agree with Coseriu (1977, p. 50), but on the basis of a bigger data-base and on the basis of a survey or more types, that "[es] gibt... aber überhaupt keine Exozentrika, sondern ausschließlich Endozentrika: Dickkopf ist ein Kompositum von genau demselben Typ wie Rotwein" [there are absolutely no exocentrics, only endocentrics: fathead is a compound of precisely the same type as blackbird; my translation LB]. An alternative to the zero-analysis given here is that these exocentrics are really cases of metonymy. Metonymy has been suggested by many as a way of analysing exocentrics (see, for example, Booij 2002, p. 143, Benczes 2006). Here I am suggesting that a possible analysis sees all exocentrics as endocentrics

pound such as aniktókardos 'open-hearted' combines compounding and derivation and belongs to the class of extended bahuvrihis".

${ }^{61} C f$. también Ralli (2013: 113): «It is important to notice that combinations of an adjective and a noun $[\mathrm{AN}]$, or two nouns $[\mathrm{N} \mathrm{N}]$, are not the only exocentric patterns which are found in SMG [Standard Modern Greek] and its dialects. Although not as frequent as those formations, a verb-noun $[\mathrm{V} \mathrm{N}]$ pattern can also be traced in certain learned or ancient-type constructions, denoting someone or something, bearing the property which is literally or metaphorically expressed by the verb-noun complex». Y los ejemplos que proporciona son: misoyinis (misógino, odiamujeres), xasoméris (pierdedías), filómusos (ama[las]musas), firóoikos (huyejuicios, fugitivo). 
interpreted by figures of speech, but not necessarily as the same figure of speech in every case (Bauer 2008: 70).

Una vez tratados los compuestos posesivos y los determinativos nos queda decir dos palabras de nuevo sobre los llamados compuestos de rección, tanto verbal como preposicional. Los primeros, que son los más numerosos con mucho tanto en griego como en español (frente a lo que ocurre en alemán, donde la inmensa mayoría son compuestos determinativos sustantivos), corresponden a nuestros compuestos morfológicos nucleoverbales, precisando que, cuando, en un compuesto, hay un elemento verbal, este es siempre el miembro determinado del compuesto, independientemente de la posición que ocupe: lo mismo sucede en griego antiguo y moderno que en español. Así, por ejemplo, en el ejemplo quevediano calvicasadas, compuesto referido a doncellas casadas con hombres calvos ${ }^{62}$, está claro que el miembro determinado es el participio que ocupa el segundo lugar. Y lo mismo pasa en arcaísmos y préstamos, como grandilocuente, fehaciente o vasodilatador.

Por lo que toca a los compuestos de rección preposicional, ya hemos dicho que, actualmente, suelen estudiarse dentro de la derivación. Sin embargo, Risch (1974²: 187-189 y, sobre todo, 1981[1944]: 106) incluye dentro de estos compuestos un subtipo muy interesante, que deriva de sintagmas y produce Ableitungskomposita (compuestos por derivación). Traducimos sus palabras ${ }^{63}$ :

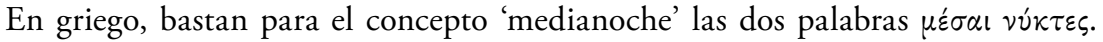
Cuando se quiere construir un adjetivo de relación se toma el sufijo habitual-105y,

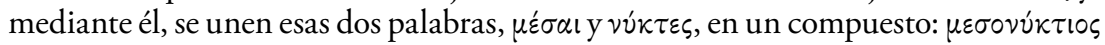
(por primera vez en Píndaro). De la misma manera que al lado de $v u ́ \xi$ está el adjetivo

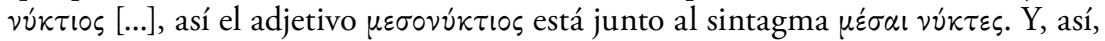
estas dos palabras forman un compuesto por medio de la derivación. La composición y la derivación tienen lugar al mismo tiempo. Por eso, aunque se puede hablar de compuesto derivativo (Ableitungskompositum), quizá sería mejor hablar de derivación

\footnotetext{
verlas difuntas».

${ }^{63}$ Literalmente: «Im Griechischen genügen also für den Begriff ,Mitternacht' die zwei

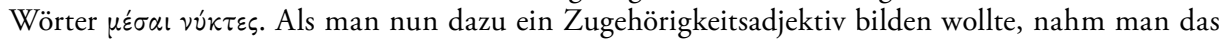

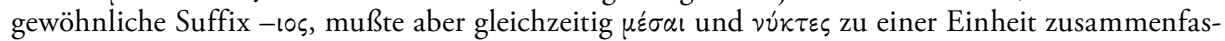

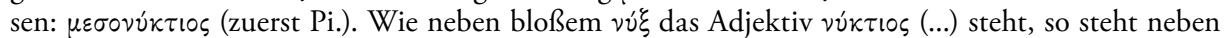

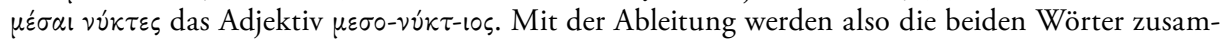
mengezogen, Komposition und Ableitung erfolgen gleichzeitig. Man kann daher von einem Ableitungskompositum sprechen, besser wäre vielleicht komponierende oder Kompositionsableitung). Was wir im Falle von $\mu \varepsilon \sigma \circ v \dot{x} \tau i \circ \varsigma$ festgestellt haben, können wir noch an zahlreichen andern Bil-

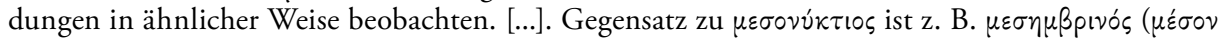

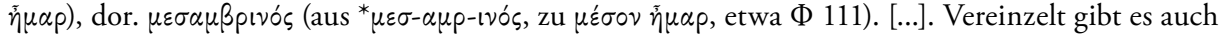
$\mu \varepsilon \sigma \eta \dot{\eta} \mu \beta$ p $о \varsigma$, während das substantivierte Femininum $\mu \varepsilon \sigma \eta \mu \beta p i \alpha$ wieder ganz geläufig ist. Weitere Bildungen mit $\mu \varepsilon \sigma \circ-$ sind etwa $\mu \varepsilon \sigma o ́ \gamma \alpha ı \varsigma$ (...) ,im Innern des Landes gelegen oder wohnend' (substantiviert $\dot{\eta} \mu \varepsilon \sigma o ́ \gamma \alpha \iota \alpha)$. [...] [I]n beiden Fällen dient also als Grundlage eine aus zwei wichtigen Wörtern bestehende Wendung, die man adverbiell bezeichnen kann».
}

${ }^{62}$ Cosa que el poeta desaconseja vivamente a sus madres: «Antes que calvicasadas / es mejor 
compositiva (komponierende oder Kompositionsableitung). Lo que hemos comprobado en el caso de $\mu \varepsilon \sigma o v \cup ́ \kappa \tau ı$, lo podemos observar también en muchos otros casos. [...]

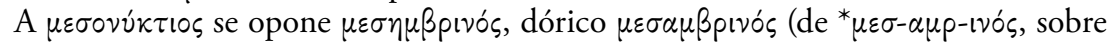

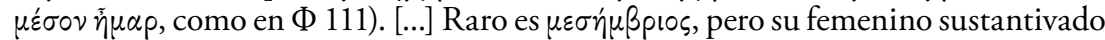
$\mu \varepsilon \sigma \eta \mu \beta$ pí $\alpha$ es muy corriente. Otra construcción con $\mu \varepsilon \sigma o-$ es $\mu \varepsilon \sigma o ́ \gamma \alpha$ เos [...] 'situado o que vive en el interior de la tierra/tierra adentro' (sustantivado en $\mu \varepsilon \sigma o ́ \gamma \alpha \iota \alpha$ ). [...] En ambos casos les sirve de base una construcción de tipo adverbial que consta esencialmente de dos palabras (Risch 1981[1945]: 114-115).

Estos compuestos serían los correspondientes a nuestros centrocampista, sietemesinos, etc., en los que no entraremos en esta ocación. Y, por otra parte, en griego antiguo se documentan también una suerte de compuestos coordinativos sustantivos, llamados Mischungskomposita (compuestos mixtos) por Risch (1981[1944]: 56-61), del tipo de nuestro baciyelmo, que, en griego, se refieren sobre todo a seres fabulosos: aquí tampoco hay relación de determinación entre sus miembros, sino que ambos están en un plano de igualdad, como ocurre con los dvandva o compuestos copulativos o coordinativos, pero, a diferencia de estos, no designan dos conceptos, sino uno solo, que viene a ser una suerte de compromiso o punto medio entre las referencias habituales de ambos miembros. A este grupo de compuestos mixtos pertenecen, por ejemplo, el i $\pi \pi \circ \alpha \dot{\alpha} v \theta$ apos 'escarabajo usado como caballo de montar' que aparece en $L a$ paz, de Aristófanes y que podríamos traducir por 'escarabajicaballo' o los I $\pi \pi \circ \gamma$ v́roıs o Buitricaballos de la Historia verdadera de Luciano de Samosata, los primeros selenitas fabulosos que encontraron Luciano y sus compañeros al alunizar, de los que el autor nos dice, sin embargo, que no eran buitres ni caballos, sino «hombres que cabalgaban sobre grandes buitres» (Batista 2016: 78-80) ${ }^{64}$.

Así pues, concluyendo podemos decir que, a pesar del muy diferente uso que hacen de la composición de palabras, tanto en alemán como en español podemos encontrar casi todos los tipos de palabras compuestas que se documentan en griego, la lengua que mayor empleo hace de ella.

\section{CONCLUSIONES}

De acuerdo con los objetivos que nos habíamos planteado, la aportación más importante de nuestro trabajo es la propuesta de una nueva clasificación de la composición nominal en español. Tras analizar críticamente las clasificaciones más extendidas aportadas para nuestra lengua en los últimos treinta años, abandonamos la línea más seguida en ellas, que toma como criterio rector de sus cla-

${ }^{64}$ En cambio, las I $\pi \pi \circ \mu \nu \dot{p} \mu \eta \kappa \varepsilon \varsigma$ u Hormiguicaballos eran hormigas, aunque gigantes, y los $\Psi \nu \lambda \lambda \circ \tau \circ \xi \delta_{\tau} \alpha \iota$ o Arqueripulgas eran arqueros que debían su nombre a que cabalgan sobre pulgas del

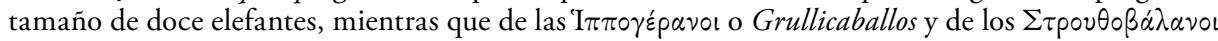
o Glandigorriones ignoramos cómo eran porque Luciano dice que no los vio y, por tanto, no se atreve a describirlos. 
sificaciones la categoría gramatical, ya sea la del compuesto o la de sus elementos constituyentes. Frente a esta tendencia, nuestra clasificación toma como eje principal la relación semántico-sintáctica que se da entre los dos miembros constituyentes de los compuestos léxicos, así como su particular reflejo formal. Aplicando dicho criterio, distinguimos, en el primer nivel, entre compuestos léxicos propios o morfológicos (pelirrojo, trotaconventos) y compuestos léxicos impropios, sintagmáticos o sintácticos (bocacalle, camposanto, buenaventura, carricoche, rojinegro). Ambos tipos resultan de la aplicación de procedimientos formativos claramente diferentes: los compuestos propios se construyen a partir de procedimientos exclusivos de formación de palabras, mientras que los compuestos impropios se forman mediante la aplicación de las mismas reglas que operan en sintaxis libre. Esta diferencia se refleja claramente en el hecho de que en los (dos tipos de) compuestos propios o morfológicos se opera siempre un cambio de categoría de la nueva palabra con respecto a su núcleo: así, en el tipo pelirrojo, el núcleo y elemento determinado del compuesto es un sustantivo mientras que el compuesto es adjetivo; y paralelamente, en el tipo trotaconventos, el núcleo y elemento determinado es un verbo, mientras que el compuesto es un adjetivo, frecuentemente sustantivado. Contrariamente a estos, los compuestos impropios o sintácticos presentan siempre la misma categoría de su núcleo: así, bocacalle es un sustantivo como su núcleo boca y camposanto un sustantivo como su núcleo campo. Obviamente, en los casos en los que la relación de los constituyentes de estos compuestos impropios sintácticos es coordinativa, no hablamos de núcleo y la categoría del compuesto es siempre la misma que la de sus dos constituyentes: así, el compuesto sintáctico coordinativo, carricoche, es un sustantivo como sus componentes carro y coche, y rojiblanco es un adjetivo como sus miembros rojo y blanco. Por otra parte, si correlacionamos los conceptos clásicos de exocentrismo y endocentrismo con el cambio de la categoría gramatical del compuesto con respecto a su núcleo, diríamos que los compuestos propios son exocéntricos, mientras que los impropios son endocéntricos. Igualmente, otra característica fundamental en la diferenciación entre estos dos grandes tipos de compuestos léxicos españoles es el hecho de que los compuestos propios siempre presentan un tema entre sus constituyentes: el tema nominal pel- en pelirrojo y el tema verbal trot (a) - en trotaconventos mientras que los compuestos impropios contienen palabras enteras como constituyentes. En este sentido, planteamos que los compuestos propios o morfológicos constituyen el dominio nuclear o prototípico dentro de la composición nominal en español, por lo que pensamos que, junto a la derivación, conforman el componente de formación de palabras de nuestra lengua. Por el contrario, los compuestos impropios o sintácticos se sitúan en un dominio periférico más cercano a la fraseología.

Por último, hemos intentado poner de manifiesto la importancia de tener presentes los aspectos diacrónicos y tipológicos en el estudio de los compuestos españoles (y románicos). Y, en nuestro caso, creemos que estas consideraciones diacrónicas y tipológicas contribuyen a avalar sólidamente los criterios clasificatorios que hemos empleado y, en consecuencia, nuestra nueva propuesta de clasificación. En este sentido, tanto en griego como en español, los compuestos son, en su mayoría, adjetivos exocéntricos o posesivos, lo cual, si bien concuerda con el carácter origi- 
nariamente adjetivo de la composición indoeuropea, no ocurre en alemán, donde la gran mayoría de los compuestos son sustantivos determinativos.

De acuerdo a lo tratado y retomando las preguntas que nos hacíamos al inicio de este trabajo, consideramos, en suma, que palabra compuesta es toda aquella unidad léxica o lexía formada por dos o más lexemas, sin que este concepto de palabra compuesta se pueda igualar con el de compuesto nominal, que es mucho más restringido. Pues, en efecto, los compuestos nominales, al igual que los derivados, son el resultado de procedimientos concretos de formación de palabras heredados del sistema latino, mientras que, entre las palabras compuestas, encontramos grupos muy heterogéneos de lexías entre las que cabe destacar, principalmente, las frases lexicalizadas (correveidile, hazmerreir, nomeolvides), los llamados compuestos sintagmáticos (mesa de noche, dinero negro, libre mercado, retrato robot), las formas cultas, arcaicas o latinizantes (ecografía, plenilunio, altitonate, grandilocuente) y distintos tipos de préstamos o calcos semánticos (trabajoadicto, piano bar, teleserie, radionovela), que, en ningún caso, responden a los mencionados procedimientos sincrónicos de formación de palabras, internos a la gramática de la lengua española y exclusivos para tal fin. 


\section{BIBLIOGRAFÍA}

Alarcos García, Emilio (1955): "Quevedo y la parodia idiomática», Archivum 5: 3-38 (https:/cvc. cervantes.es/literatura/quevedo_critica/satiras/alarcos.htm\#np1).

Aleksandrow, Aleksander (1888): Litauische Studien: I. Nominalzusammensetzungen, Dorpat: Hermann (http://dspace.ut.ee/bitstream/handle/10062/4632/aleksandrowlita uischeocr. pdf? sequence $=4 \&$ isAllowed $=y$ ).

Almela, Ramón (1999): Procedimientos de formación de palabras en español, Madrid: Cátedra.

Andreou, Marios (2014): Headedness in Word Formation and Lexical Semantics: evidence from Italiot and Cypriot, tesis defendida en la Universidad de Patras (Grecia) http://nemertes.lis.upatras.gr/jspui/bitstream/10889/8466/1/Andreou_Thesis.pdf.

Andreou, Marios y Angela Ralli (2015): «Form and Meaning of Bahuvrihi Compounds: Evidence from Modern Greek and Its Dialects», en Laurie Bauer, Lívia Körtvélyessy y Pavol Štekauer (eds.), Semantics of Complex Words, Cham et al.: Springer, 163-185.

BADER, Françoise (1962): La formation des composés nominaux du latin, Paris: Les Belles Lettres.

BaIst, Gottfried (1899): «Longimanus und manilargo», Romanische Forschungen 10: 471-474.

Batista, José Juan (1988): Composición de palabras en la épica griega arcaica, La Laguna: Servicio de publicaciones de la Universidad de La Laguna (en microfichas).

Batista, José Juan (2016): «De nuevo sobre composición de palabras en griego y español: a propósito de las Verae Historiae de Luciano", en Elena Redondo Moyano y María José García Soler (eds.), Nuevas interpretaciones del Mundo Antiguo, Vitoria: Universidad del País Vasco, 67-86.

Batista, José Juan, Aitor Mora y Juan Manuel Pérez Vigaray (2019): «Clasificación y exocentricidad en compuestos griegos, alemanes y españoles», en Ana Díaz Galán y Marcial Morera (eds.), Nuevos estudios de lingüistica moderna, Frankfurt et al.: Peter Lang, 81-97.

Bauer, Laurie (2008): «Exocentric compounds», Morphology 18: 51-74.

Bauer, Laurie (2017): Compounds and Compounding, Cambridge: Cambridge University Press.

Benveniste, Émile (1977 [1974]): «Fundamentos sintácticos de la composición nominal», en Problemas de lingüistica general, Madrid: Siglo XXI, tomo II, 147-163.

Bisetto, Antonia y Sergio Scalise, (2005): «The classification of compounds», Lingue e Linguaggio 4: 319-332 (http://www.morbocomp.sslmit.unibo.it/ download/classification_of_compounds.pdf).

Bloomfield, Leonard (1933): Language, New York: Henry Holt \& Co.

BooIj, Geert (2005): «Compounding and derivation: evidence for Construction Morphology», en Wolfgang Dressler, Dieter Kastovsky, Oskar Pfeiffer y Franz Rainer (eds.), Morphology and its demarcations: Selected papers from the 11th Morphology meeting, Amsterdam: John Benjamins, 109-132 (https://geertbooij.files.Wordpress. com/2014/02/booij-2005-compounding-and-derivation.pdf).

Boolj, Geert (2012³): The Grammar of Words, Oxford: OUP.

Bopp, Franz (1827): Ausführliches Lehrgebäude der Sanskrita-Sprache, Berlin: Dümmler.

Bopp, Franz (1852): Vergleichende Grammatik des Sanskrit, Zend, Griechischen, Lateinischen, Litthauischen, Altslawischen, Gothischen und Deutschen, Sechste Abtheilung, Berlin: Dümmler. 
Bork, Hans Dieter (1990): Die lateinisch-romanischen Zusammensetzungen Nomen + Verb und der Ursprung der romanischen Verb-Ergänzung-Komposita, Bonn: Romanistischer Verlag.

Brugmann, Karl (1905-1906): "Zur Wortzusammensetzung in den indogermanischen Sprachen», Indogermanische Forschungen 18: 59-76.

Brugmann, Karl (1906²): Grundriss der vergleichenden Grammatik der indogermanischen Sprachen, Strassburg: Trübner (tomo II, 1. ${ }^{a}$ parte).

Buck, Carl Darling (1933): Comparative Grammar of Greek and Latin, Chicago: University of Chicago Press.

Buenafuentes de la MATA, Cristina (2001-2002): «Procesos de gramaticalización en el estudio de los compuestos del español: el caso del elemento malla(-)", Anuari de Filologia 11-12: 17-29.

Buenafuentes de la mata, Cristina (2007): Procesos de gramaticalización y lexicalización en la formación de compuestos en español, tesis defendida en la Universidad Autónoma de Barcelona y accesible en https://www.tdx.cat/handle/10803/4879.

Buenafuentes de la Mata, Cristina (2009): «La formación de palabras compuestas: del latín al español», en Joan Rafel Cufi (ed.), Diachronic Linguistics, Gerona: Documenta Universitaria, 213-238.

BüHLER, Karl (1979[1934]): Teoría del lenguaje, Madrid: Alianza Editorial.

Bustos Gisbert, Eugenio (1986): La composición nominal en español, Salamanca: Universidad de Salamanca.

Coseriu, Eugenio (1977): Estudios de lingüistica románica, Madrid: Gredos.

Coseriu, Eugenio (1978): «La formación de palabras desde el punto de vista del contenido (a propósito del tipo "coupe-papier"”, en Eugenio Coseriu, Gramática, semántica, universales, Madrid: Gredos, 239-264.

Coseriu, Eugenio (2003 [1982]): «Los procedimientos semánticos en la formación de palabras», Odisea 3: 179-189.

Debrunner, Albert (1917): Griechische Wortbildungslehre, Heidelberg: Carl Winters.

FÁbregas, Antonio (2015): La Morfología. El análisis de la palabra compleja, Madrid: Síntesis.

Felíu Arquiola, Elena (2008): «Palabras con estructura interna», en Elena de Miguel (ed.), Panorama de la lexicología, Barcelona: Ariel, 51-82.

García Lozano, Francisco (1993 [1978]): «Los compuestos de sustantivo + adjetivo del tipo pelirrojo», en Soledad Varela (ed.), La formación de palabras, Madrid: Taurus, 205-214.

García Padrón, Dolores y José Juan Batista (2010): «Las combinaciones nominales en español: aspectos sintácticos, semántico-denotativos y terminológicos", LEA (Lingüistica española actual) 32, 2: 197-222.

Gather, Andreas (2001): Romanische Verb-Nomen-Komposita, Tübingen: Narr.

GAUger, Hans-Martin (1971): Untersuchungen zur spanischen und französischen Wortbildung, Heidelberg: Carl Winter.

Giammatteo, Mabel y Augusto Trombetta (2015): "Quitapenas, abrelatas y rompecorazones. Formación e interpretación de los compuestos de "verbo + nombre" en español», Boletín de Filología 50, 1: 195-208. 
Granvik, Anton (en prensa): «La preposición de como posible marca de subordinación», en Rafael García Pérez y Yuko Morimoto (eds.), De la oración al discurso: estudios en español y estudios contrastivos, Frankfurt am Main: Peter Lang.

Hernández Paricio, Francisco y Bárbara Marqueta Gracia (2015): «Lexicalización de esquemas compositivos argumentales», Anuario de Lingüistica Hispánica 30: 119-143.

Hinojo Andrés, Gregorio (2003): “CCazadotes”: ¿Latino o románico?», en Fernando Sánchez Miret (ed.), Actas del XXIII Congreso Internacional de Lingüistica y Filología Románica, Tübingen: Niemeyer, tomo I, 357-369.

Hinojo Andrés, Gregorio (2009): «La utilización del léxico griego en la Cena Trimalchionis», Faventia 31: 71-86.

JimÉnez Ríos, Enrique (1999): "Aguafiestas, metepatas y otros compuestos nominales peculiares», Estudios de Lingüistica 13, 117-148.

LANG, Mervin F. (1992): Formación de palabras en español. Morfología derivativa productiva en el léxico moderno, Madrid: Cátedra.

Lieber, Rochelle y Pavel Štekauer (eds.) (2009): The Oxford Handbook of Compounding, Oxford: OUP.

Lindner, Thomas (2011): «Komposition», en Thomas Lindner (ed.), Indogermanische Grammatik, Heidelberg: Winter, tomo Iv/1, 1 .r $^{\text {er }}$ cuadernillo.

Lloyd, Paul (1968): Verb-Complement Compounds in Spanish, Tübingen: Max Niemeyer.

LÜDTKE, Jens (2006): «Probleme einer funktionellen romanischen Wortbildungslehre: gibt es „Parasynthese“?», en Carmen Kelling, Judith Meinschaefer \& Katrin Mutz (eds.), Morphologie und romanistische Sprachwissenschaft, Konstanz: Universität Konstanz, 125-139 (http:// kops.ub.uni-konstanz.de/volltexte/2006/1812/pdf/AP_120.pdf).

LÜDTKE, Jens (2011): La formación de palabras en las lenguas románicas. Su semántica en diacronía y sincronía, México: El Colegio de México.

Manolessou, Ioanna y Angela Ralli (2015): «From Ancient Greek to Modern Greek», en Peter Müller, Ingeborg Ohnheiser, Susan Olsen y Franz Rainer (eds.), Word-Formation. An International Handbook of the Languages of Europe, Berlin: De Gruyter / Mouton, vol. 3, 2041-2061.

Martinet, André (1969): «La palabra», en Émile Benveniste et al:: Problemas del lenguaje, Buenos Aires: Editorial Sudamericana, 37-51.

Meillet, Antoine y Joseph Vendryes, (19795): Traité de grammaire comparée des langues classiques, Paris: Honoré Champion.

Meissner, Torsten y Olaga Tribulato (2002): «Nominal Composition in Mycenaean Greek», Transactions of the Philological Society 100, 3: 289-330

Meunier, Louis-Francis (1872): Études de grammaire comparée. Les composés syntactiques en grec, en latin, en français et subsidiairiament en zend et en indien, Paris: Durand et Pedone-Lauriel.

Meunier, Louis-Francis (1875): Les composés qui contiennent un verbe à un mode personnel en latin, en français, en italien et en espagnol, Paris: L'Imprimerie Nationale.

Montes Giraldo, José Joaquín (1977): «Un tipo de composición nominal y el "español atlántico”", Thesaurus 23, 3: 653-659. 
Montoro del Arco, Esteban (2008): «Relaciones entre Morfología y Fraseología: las formaciones nominales pluriverbales», en Esteban Tomás Montoro del Arco y Ramón Almela Pérez (eds.), Neologismo y morfologia, Murcia: Universidad de Murcia, 121-146.

Morciniec, Norbert (1964): Die nominalen Wortzusammensetzungen in den westgermanischen Sprachen, Wrocław: Zakład.

Morera, Marcial (1998): «Sobre los nombres compuestos con el formante -i-», Revista de Filología de la Universidad de La Laguna 15: 167-176.

Morera, Marcial (2005): La complementación morfológica en español, Frankfurt am Main et al.: Peter Lang.

Moyna, María Irene (2011): Compound Words in Spanish. Theory and History, Amsterdam / Philadelphia: John Benjamins.

Munthe, Åke (1889): «Observations sur les composés espagnols du type aliabierto», en Recueil de mémoires philologiques présenté à M. Gaston Paris par ses éleves suédois, Stockholm: L'Imprimerie Centrale, 31-56.

Munthe, Åke (1901): «Bemerkungen zu Baists schrift "Longimanus und manilargo"», en Uppsatser $i$ romansk filologi tillägnade Professor Per Adolf Geijer på hans sextioårsdag, Uppsala: Almqvist \& Wiksells, 57-72.

Neckel, Gustav (1906): «Exozentrische Komposition», Indogermanische Forschungen 19: 249-254.

NoordegraAf, Jan (1989): «From the history of the term 'exocentric'», Historiographia Linguistica 16: 211-215 (https://core.ac.uk/download/pdf/15452465.pdf).

Nord, Christiane (1983): Neueste Entwicklung im spanischen Wortschatz, Rheinfelden: Schäuble.

Oniga, Renato (1988): I composti nominali latini, Bologna: Pàtron.

Oniga, Renato (1992): "Compounding in Latin», Morphology of Compounding, Rivista di Linguistica 4: 97-116.

Pérez Vigaray, Juan Manuel (2004 [1994]): La composición nominal en español, Las Palmas: Universidad de Las Palmas: https://accedacris.ulpgc.es/handle/10553/2242.

Pérez Vigaray, Juan Manuel y José Juan Batista (2002): «Apuntes sobre el infijo /i/ en la composición nominal del español», en Sybille Große y Axel Schönberger (eds.), Ex oriente lux. Festschrift für Eberhard Gärtner zu seinem 60. Geburtstag, Frankfurt am Main: Valentia, 341-349.

Pérez Vigaray, Juan Manuel y José Juan Batista (2005): "Composición nominal y fraseología», en Ramón Almela, Gerd Wotjak y Estanislao Ramón Trives (coords.), Fraseología contrastiva: con ejemplos tomados del alemán, español, francés e italiano, 81-90.

Petersen, Walter (1914-1915): «Der Ursprung der Exozentrika», Indogermanische Forschungen 34: 254-285.

Pinker, Steven (1995): El instinto del lenguaje, Madrid: Alianza.

Pottier, Bernard (1972²): Presentación de la lingüistica, Madrid: Alcalá.

RaINer, Franz (1993): Spanische Wortbildungslehre, Tübingen: Niemeyer.

Rainer, Franz y Soledad Varela (1992): "Compounding in Spanish», Rivista di Lingüistica 4: 117-142.

Ralli, Angela (2009): «IE, Hellenic: Modern Greek», en Rochelle Lieber y Pavol ŠTeKauer: The Oxford Handbook of Compounding, Oxford: OUP, 453-463.

RaLLI, Angela (2009a): «Modern Greek dvandva compounds: a linguistic innovation in the history of Indo-European languages», Word Structure 2: 48-68. 
Ralli, Angela (2013): Compounding in Modern Greek, Dordrecht: Springer.

Ralli, Angela y Marios Andreou (2012): «Revisiting Exocentricity in Compounding: Evidence from Greek and Cypriot», en Ferenc Kiefer et al. (eds.), Current Issues in Morphological Theory: (Ir) regularity, analogy and frecuency, Amsterdam: John Benjamins, 65-81.

Real Academia Española (2010): Nueva gramática de la lengua española, Madrid: Espasa.

RICCA, Davide (2005): «Al limite tra sintassi e morfologia: i composti aggettivali V-N nell' italiano contemporaneo», en Maria Grossmann \& Anna Thornton (eds.), La formazione delle parole, Roma: Bulzoni, 465-486.

Ricca, Davide (2010): «Corpus data and theoretical implications with special reference to Italian V-N compounds», en Sergio Scalise e Irene Vogel (eds.), Cross-disciplinary Issues in Compounding, Amsterdam: John Benjamins, 237-254.

Risch, Ernst (1974²): Wortbildung der homerischen Sprache, Berlin: de Gruyter.

Risch, Ernst (1981): Kleine Schriften, edición preparada por Annemarie Etter et alii, Berlín / New York: Walter De Gruyter.

Rohrer, Christian (1977 [1965]): Die Wortzusammensetzung im modernen Französisch, Tübingen: Narr.

SÁNCHez, Juan (2009): «La formación de palabras por composición desde un punto de vista histórico", Revista de Filología Español Lxxxıx: 103-128.

Scalise, Sergio, Antonio Fábregas y Francesca Forza (2009): «Exocentricity in Compounding», Gengo Kenkyu 135: 49-84.

Schwyzer, Eduard (19906 [1939]): Griechische Grammatik: Allgemeiner Teil, Lautlehre, Wortbildung, Flexion, München: Beck.

Tabares, Encarna y José Juan Batista (2013): «Los compuestos verbonominales en español: ¿sustantivos o adjetivos?», Zeitschrift für romanische Philologie 129: 652-672.

Tabares, Encarna, Juan Manuel Pérez Vigaray y José Juan Batista (2012): «Los compuestos españoles del tipo de trotaconventos», en Gerd Wotjak, Dolores García Padrón y María del Carmen Fumero Pérez (eds.), Estudios sobre lengua, cultura y cognición, Frankfurt am Main et al:: Peter Lang, 237-248.

Tribulato, Olga (2006): "Homeric $\theta \nu \mu \nu \lambda \varepsilon ́ \omega \nu$ and the Question of Greek "Reversed Bahuvrīhis"», Oxford University Working Papers in Linguistics, Philology and Phonetics 11: 162-178.

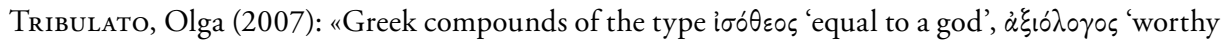

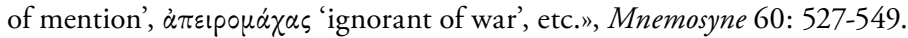

Tribulato, Olga (2015): Ancient Greek Verb-Initial Compounds: Their Diachronic Development Within the Greek Compound System, Berlin/Boston: De Gruyter.

UHLICH, Jürgen (1997): «Der Kompositionstyp „Armstrong“ in den indogermanischen Sprachen», Historische Sprachforschungen 110, 1: 21-46.

VAL Álvaro, José Francisco (1999): «La composición», en Ignacio Bosque y Violeta Demonte (eds.), Gramática descriptiva de la lengua española, Madrid: RAE/Espasa-Calpe, tomo III, 47574841.

VArela, Soledad (1990a): Fundamentos de morfología, Madrid: Síntesis.

VArela, Soledad (1990b): «Composición nominal y estructura temática», Revista Española de Lingüística 20: 55-81.

VArela, Soledad (2005): Morfología léxica: la formación de palabras, Madrid: Gredos. 
Whitehead, Benedicte Nielsen (2013): “Bahuvrihi”, possessive and exocentric compounds: Right and wrong turns from India to the West (poster)», en http://www. academia. edu/4507824/_ Bahuvrihi_possessive_and_exocentric_compounds_right_and_wrong _turns_from_ India_to_the_west_poster_.

ZaCarías Ponce de León, Ramón Felipe (2018): «Tipos de predicación metonímica en la composición nominal del español de México", Cuadernos de Lingüistica de El Colegio de México 5, accesible en https://cuadernoslinguistica.colmex.mx/index.php/cl/article/view/117/136. 
